\title{
An attribute-diversity approach to functional diversity, functional beta diversity, and related (dis)similarity measures
}

\author{
Anne Chao,,${ }^{1,8}$ Chun-Huo Chiu, ${ }^{2}$ Sébastien Villéger, ${ }^{3}$ I-Fang Sun, ${ }^{4}$ Simon Thorn, ${ }^{5}$ Yi-Ching Lin, ${ }^{6}$ \\ Jyh-Min Chiang, ${ }^{6}$ And William B. Sherwin ${ }^{7}$ \\ ${ }^{1}$ Institute of Statistics, National Tsing Hua University, Hsin-Chu 30043 Taiwan \\ ${ }^{2}$ Department of Agronomy, National Taiwan University, Taipei 10617 Taiwan \\ ${ }^{3}$ MARBEC, Université de Montpellier, CNRS, IFREMER, IRD, Place Eugène Bataillon, 34095 Montpellier, France \\ ${ }^{4}$ Department of Natural Resources and Environmental Studies, National Tong Hwa University, Hualien 97401 Taiwan \\ ${ }^{5}$ Field Station Fabrikschleichach, Biocenter, University of Würzburg, Glashüttenstrasse 5, 96181 Rauhenebrach, Germany \\ ${ }^{6}$ Department of Life Science, Tunghai University, Taichung 40704 Taiwan \\ ${ }^{7}$ Evolution \& Ecology Research Centre, School of Biological Earth and Environmental Science, The University of New South Wales, \\ Sydney, New South Wales 2052 Australia
}

Citation: A. Chao, C.-H. Chiu, S. Villéger, I-F. Sun, S. Thorn, Y.-C. Lin, J.-M. Chiang, and W. B. Sherwin. 2019. An attribute-diversity approach to functional diversity, functional beta diversity, and related (dis)similarity measures. Ecological Monographs 89(2):e01343. 10.1002/ ecm. 1343

Abstract. Based on the framework of attribute diversity (a generalization of Hill numbers of order $q$ ), we develop a class of functional diversity measures sensitive not only to species abundances but also to trait-based species-pairwise functional distances. The new method refines and improves on the conventional species-equivalent approach in three areas: (1) the conventional method often gives similar values (close to unity) to assemblages with contrasting levels of functional diversity; (2) when a distance metric is unbounded, the conventional functional diversity depends on the presence/absence of other assemblages in the study; (3) in partitioning functional gamma diversity into alpha and beta components, the conventional gamma is sometimes less than alpha. To resolve these issues, we add to the attribute-diversity framework a novel concept: $\tau$, the threshold of functional distinctiveness between any two species; here, $\tau$ can be chosen to be any positive value. Any two species with functional distance $\geq \tau$ are treated as functionally equally distinct. Our functional diversity quantifies the effective number of functionally equally distinct species (or "virtual functional groups") with all pairwise distances at least $\tau$ for different species pairs. We advocate the use of two complementary diversity profiles ( $\tau$ profile and $q$ profile), which depict functional diversity with varying levels of $\tau$ and $q$, respectively. Both the conventional species-equivalent method (i.e., $\tau$ is the maximum of species-pairwise distances) and classic taxonomic diversity (i.e., $\tau$ is the minimum of non-zero species-pairwise distances) are incorporated into our proposed $\tau$ profile for an assemblage. For any type of species-pairwise distance matrices, our attribute-diversity approach allows proper diversity partitioning, with the desired property gamma $\geq$ alpha and thus avoids all the restrictions that apply to the conventional diversity decomposition. Our functional alpha and gamma are interpreted as the effective numbers of functionally equally distinct species, respectively, in an assemblage and in the pooled assemblage, while beta is the effective number of equally large assemblages with no shared species and all species in the assemblages being equally distinct. The resulting beta diversity can be transformed to obtain abundance-sensitive Sørensen- and Jaccard-type functional (dis)similarity profiles. Hypothetical and real examples are used to illustrate the framework. Online software and R codes are available to facilitate computations.

Key words: attribute diversity; diversity decomposition; functional (dis) similarity; functional beta diversity; functional diversity; Hill numbers; quadratic entropy; species diversity; species traits; trait diversity.

\section{INTRODUCTION}

Functional diversity, widely regarded as a key to understanding ecosystem processes and response to environmental stress or disturbance (Tilman et al. 1997,

Manuscript received 9 January 2018; revised 11 September 2018; accepted 27 September 2018. Corresponding Editor: Helmut Hillebrand.

${ }^{8}$ E-mail: chao@stat.nthu.edu.tw
Cadotte et al. 2011, Naeem et al. 2012) is typically quantified by using measures based on species trait values and any measure of species abundance/dominance. Functional diversity has been used extensively for the last decade to address diverse ecological questions such as highlighting biogeographical patterns (e.g., Violle et al. 2014), assessing its response to assembly processes along environmental gradients (e.g., Villéger et al. 2012) and testing its effect on ecosystem functioning (e.g., Tilman 
et al. 1997, Hooper et al. 2005, Cadotte et al. 2011, Mouillot et al. 2011). The resolution of the above questions relies on robust and informative measures that can properly assess not only within-assemblage functional diversity but also among-assemblage differentiation.

Functional diversity is not expressible as a single number or just a few measures; an enormous number of measures have been proposed to quantify the complementary aspects of trait value distribution among species (Mouchet et al. 2010, Weiher 2011, Swenson et al. 2012, Mason et al. 2013, Mouillot et al. 2013, Scheiner et al. 2017). There is no consensus about how to quantify and compare functional diversity among multiple assemblages. Different perspectives have led to measures that differently quantify the diversity of species trait values accounting for species abundance/dominance. The responses of species to environmental drivers and/or the effect of species on ecosystem processes are likely to be contrastingly affected by the relative role of their trait values and of their dominance in assemblages. Therefore, it is crucial to develop a set of indices that measure the complementary facets of functional diversity incorporating both species abundances and trait values.

There are three major approaches to calculating functional diversity: trait-value based, dendrogram based, and distance based. For the trait-value-based approach, measures are calculated from species trait values directly (e.g., aggregated-trait value; Garnier et al. 2004), or by accounting for their position in a multidimensional functional space built according to their trait values (e.g., volume occupied by species; Cornwell et al. 2006, Mouillot et al. 2013). In the dendrogram-based approach (Petchey and Gaston 2002), a functional dendrogram is constructed by applying a clustering algorithm to the species pairwise distance matrix. However, it has been shown that different clustering methods may lead to different conclusions (Mouchet et al. 2008) and, more recently, that even the best dendrogram is often of very low quality (Maire et al. 2015). The distance-based approaches are constructed based on the functional pairwise distance matrix computed on a set of several traits and do not require dendrograms. Mason et al. (2005) and Villéger et al. (2008) have proposed that trait-based functional diversity should include three primary components: functional richness, functional evenness, and functional divergence. In this paper, we focus on the distance-based approach and propose functional diversity measures that can be used to quantify the three functional components.

Rao's (1982) quadratic entropy $Q$, which accounts for both functional distances and species abundances, has been widely used in quantifying functional diversity. However, like its ancestral measure (Gini-Simpson index), $Q$ does not obey the replication principle, introducing interpretational problems in ecological applications if $Q$ is directly used for measuring functional diversity; see Ricotta and Acosta (2014) and the examples given in Chao et al. (2010: their Supplement). Here, the replication principle for taxonomic diversity translates as follows: given $N$ equally large (i.e., equally weighted) and equally diverse assemblages with no species in common, the diversity of the pooled assemblage will be $N$ times the diversity within a single assemblage. Ricotta and Szeidl (2006) were the first to extend $Q$ to a class of distancebased Tsallis-type entropy using a normalized distance matrix; the quadratic entropy corresponds to the distance-based Tsallis-type entropy of order 2. Their work provided the foundation for subsequent development of a general class of measures parameterized by a diversity order $q \geq 0$. However, like $Q$, distance-based Tsallis-type entropy does not satisfy the replication principle and suffers from the same problems as quadratic entropy.

Ricotta and Szeidl (2009) and de Bello et al. (2010) made an advance by converting $Q$ to "the equivalent number of maximally distinct species," which satisfies a functional version of the replication principle. Leinster and Cobbold (2012) converted the distance-based Tsallis-type entropy to its species-equivalent formulas for any diversity order $q \geq 0$; their measures are expressed in terms of similarity, rather than distance, requiring a transformation from species pairwise distance to pairwise similarity. When species similarity can be properly defined, their measures extend the concept of maximal distinction of Ricotta and Szeidl (2009) to a class of measures of any order $q \geq 0$. Throughout this paper, we will label approaches and measures that quantify the effective number of maximally distinct species as conventional species-equivalent approaches and measures; mathematical expressions are given later. For the above conventional approaches, there are three areas that could be extended and improved.

1) The conventional functional diversity is weakly sensitive to species abundances and species pairwise distances especially for species-rich assemblages; it often yields similar values (close to unity) for assemblages with contrasting levels of functional diversity. Devictor et al. (2010), Villéger et al. (2012), Stuart-Smith et al. (2013), Chao et al. (2014a), and Botta-Dukát (2018), among others, have found that when the conventional approach is applied to real data sets, very low values (near 1 or 2 , rarely exceeding 3 ) are generally obtained, especially in functional studies in which there are many $(>30)$ species and when species-pairwise distances are computed from Gower mixed-variables distances (Gower 1971). Ecologists have been inclined to conclude that a few species (one or two) are dominant in abundance and that these dominant species are functionally fairly similar. This interpretation is undoubtedly true for some data sets (e.g., Villéger et al. 2012); however, we show here that the phenomenon persists even when all species have the same abundance or when dominant species are functionally dissimilar. Thus, the conventional species-equivalent measure is generally less sensitive to the functional distance matrix and species abundance structures in real-world cases where very few species pairs are highly functionally distinct because many (i.e., >30) species are described using a few (i.e., <10) traits or functional axes. In this paper, we provide a mathematical perspective to elucidate why the conventional 
approach, based on the concept of maximal differentiation between any two species, is an almost inescapable mathematical consequence and necessarily yields low values.

2) When there is no upper bound for a distance metric (such as the commonly used Euclidean distance for continuous traits), each distance is typically normalized by dividing it with the global maximum species-pairwise distance in the pooled assemblage to obtain the conventional species-equivalent numbers; this global maximum distance depends on the assemblages involved in the study. Consequently, the conventional functional diversity of an assemblage depends on the presence/absence of other assemblages, i.e., depends on whether other assemblages are considered or not. See Conclusion and discussion for an example and details.

3) The conventional approach sometimes fails to decompose functional gamma diversity into alpha and beta components with desirable properties. When there are multiple assemblages, a critical condition for gamma quadratic entropy to always be greater than or equal to alpha quadratic entropy is that the species-pairwise distance matrix $\left(d_{i j}\right)^{1 / 2}$ must be Euclidean (Champely and Chessel 2002). This means that the conventional speciesequivalent approach sometimes fails based on quadratic entropy decomposition, giving a negative additive beta component based on $Q$, or else a multiplicative beta component (supposedly quantifying the effective number of assemblages) less than unity. For Leinster and Cobbold's (2012) measures of a general order $q$, to our knowledge, there has been no diversity decomposition in terms of alpha, beta, and gamma such that gamma is never less than alpha; see Appendix 1 of Chao et al. (2014a).

Based on the framework of attribute diversity (Chao et al. 2014a), we here develop species-equivalent formulas that refine the conventional approach, exhibit a variety of innovations, and that also avoid the three problems above. That is, (1) our measures are sensitive not only to species abundances but also to species-pairwise distances; (2) normalization of distances is not needed, and thus, our functional diversity of an assemblage is not affected by the presence/absence of other assemblages; and (3) our approach facilitates proper decomposition with desirable properties. Our approach is analogous to the proposal of Chao et al. (2010) that a time parameter $T$ should be considered in quantifying the phylogenetic diversity for a time period from $T$ years ago to the present; any two species with cophenetic distance $\geq T$ are treated as phylogenetically equally divergent and in different lineages. We here propose a corresponding parameter for functional diversity that specifies the level of threshold distinctiveness (or threshold level), denoted as $\tau$, between any two species.

Although our approach to functional diversity is grounded in an "effective" sense, we can intuitively explain the motivation for introducing the level of threshold distinctiveness from the perspective of a clustering algorithm: imagine that all species are placed in a functional space with specified pairwise distances, and now we cluster them into "virtual functional groups." As in most statistical clustering algorithm, one must first determine a threshold level such that any two species with distance greater than or equal to the specified threshold level are in different clusters and vice versa. In our framework, the threshold level $\tau$ can be chosen to be any positive value, allowing the researcher to specify that any two species with distance beyond this specified level are regarded as functionally equally distinct species and in different functional groups. Moreover, we will later show that to avoid the three problems listed above, it is imperative to introduce this parameter. When the distance of two species is less than $\tau$, we assume that only a proportion of the individuals of one species are functionally equally distinct from the other species at the threshold level $\tau$. Then for any given threshold level, we can formulate our functional diversity as a special case of the attribute diversity, i.e., a generalization of Hill numbers (Chao et al. 2014a) that can incorporate species relevant characteristics.

Our attribute-diversity approach leads to a class of distance-based functional diversity measures parameterized by a diversity order $q$. More precisely, under a specified threshold distinctiveness $\tau>0$, our proposed class of functional diversity quantifies the effective number of functionally equally distinct species (or "virtual functional groups") at the threshold level $\tau$ (i.e., all pairwise distances for different-species pairs are at least $\tau$ ). Throughout the paper, in our interpretation of effective numbers, the terms "species (equivalent)" and "(virtual) functional group" are equivalent and used interchangeably for presentation purposes.

Since all measures are expressed as functions in terms of diversity order $q \geq 0$ and the threshold distinctiveness level $\tau>0$, our functional diversity is thus characterized by a three-dimensional surface or profile that depicts the functional diversity as a function of $q$ and $\tau$. However, when there are many assemblages, so that comparison of several three-dimension surfaces becomes complicated, we show below how to use two complementary functional diversity profiles/plots to compare within-assemblage-diversity across assemblages. The first profile/plot ( $\tau$ profile) depicts the number of species-equivalents with respect to the level of threshold distinctiveness for a given diversity order $q$. The second profile/plot ( $q$ profile) depicts the number of species-equivalents with respect to the diversity order $q$ for a specified threshold distinctiveness $\tau$. When $\tau$ is specified to be the minimum of positive species-pairwise distances, the $q$ profile reduces to the species/taxonomic diversity (Hill numbers) profile; when $\tau$ is specified to be the maximum of species-pairwise distances, the $q$ profile reduces to that based on the conventional species-equivalent approach. In this way, both the conventional functional diversity and the taxonomic diversity are incorporated into the proposed $\tau$ profile. Our approach thus generalizes taxonomic diversity to include pairwise functional distances and also extends the conventional species-equivalent method to quantify the effective number of functionally equally distinct (including maximally distinct) species at any level of threshold distinctiveness. 
In addition to the assessment of within-assemblage alpha diversity, the comparison of beta/dissimilarity among assemblages is essential in quantifying effects of environmental gradients (Villéger et al. 2012) and biodiversity change (Hillebrand et al. 2018). When there are multiple assemblages, our attribute-diversity approach facilitates a proper partitioning theoretical basis from which to decompose the functional diversity of the pooled assemblage (gamma) multiplicatively into alpha and beta components for any order $q \geq 0$ and any chosen level of threshold distinctiveness $\tau>0$. As in taxonomic diversity measures, alpha and gamma are interpreted as the effective numbers of functionally equally distinct species in an assemblage and in the pooled assemblage, respectively; here "equally distinct" means that the pairwise distance between any two different-species pairs in the reference assemblage is at least $\tau$.

In the work presented here, functional gamma is never less than the alpha component; the beta component is always between the minimum value of unity (when all assemblages are functionally identical, as defined later) and the maximum value of $N$ (when there are no shared species among the $N$ assemblages and a distance between any two species from two different assemblages exceeds the threshold distinctiveness level). Our functional beta diversity thus can be interpreted as the effective number of equally large assemblages with no shared species among the assemblages and a distance between any two species of at least $\tau$. The resulting beta diversity can be further transformed to obtain the abundance-sensitive Sørensen- and Jaccard-type functional (dis)similarity profiles. We applied our functional diversity measures and decomposition to two real examples: saproxylic beetle data of three bark treatments and woody plant data based on two forest plots. These applications demonstrate that our measures can offer insights that the conventional approach cannot. A glossary and notation are provided in Appendix S1. To facilitate all computations, online software FunD (Functional Diversity) is available online and $\mathrm{R}$ codes are provided in Github (https://chao. shinyapp.io/FunD/; https://github.com/AnneChao).

\section{Ordinary Hill Numbers}

Our general framework is based on ordinary Hill numbers and their generalizations called attribute diversity, so we first present a brief outline of Hill numbers. Assume $S$ species in an assemblage, indexed by $i=1,2, \ldots, S$; let $n_{i}$ represent the raw abundance (number of individuals) of species $i$, but it could be any other metrics of species dominance, such as the relative biomass, coverage of plants or corals, or basal area of plants. The total number of individuals in the assemblage, or assemblage size, is expressed as $n_{+}=\sum_{i=1}^{S} n_{i}$. Here, $p_{i}=n_{i} / n_{+}$denotes the relative abundance of the individuals of species $i$ in the assemblage, $\sum_{i=1}^{S} p_{i}=1$. Hill (1973) proposed a class of diversity measures later called Hill numbers, or effective numbers of species, defined as the following function of species relative abundances:

$$
{ }^{q} D=\left(\sum_{i=1}^{S}\left(n_{i} / n_{+}\right)^{q}\right)^{1 /(1-q)}=\left(\sum_{i=1}^{S} p_{i}^{q}\right)^{1 /(1-q)}
$$

A consensus is emerging on the use of Hill numbers as the class of species diversity measure of choice (Ellison 2010). The parameter $q$ determines the sensitivity of the measure to the relative abundance of species. When $q=0,{ }^{0} \mathrm{D}$ is simply species richness, which counts species equally without regard to their relative abundances. For $q=1$, Eq. 1 a is undefined, but its limit as $q$ tends to 1 is the exponential of the Shannon index, referred to as Shannon diversity (Chao et al. 2014b)

$$
\begin{aligned}
{ }^{1} D=\lim _{q \rightarrow 1}{ }^{q} D & =\exp \left(-\sum_{i=1}^{S}\left(n_{i} / n_{+}\right) \log \left(n_{i} / n_{+}\right)\right) \\
& =\exp \left(-\sum_{i=1}^{S} p_{i} \log p_{i}\right) .
\end{aligned}
$$

The measure for $q=1$ counts individuals equally and weighs each species in proportion to its abundances. The measure for $q=2$, referred to as Simpson diversity, disproportionately favors dominant species.

Since Hill numbers for all $q \geq 0$ are in the same units of "species-equivalents," Hill (1973) and subsequent followers suggested that biologists present a continuous profile, a plot of Hill numbers as a function of $q \geq 0$, to convey all the information in the species relative abundance distribution. This profile, referred to as the $q$ profile, makes it easy to visually compare the diversities of multiple assemblages, and the slope of the profile can be used to judge the unevenness of the relative abundances of different species. The more uneven the species relative abundances, the more steeply the profile declines.

\section{Attribute-Diversity Approach to Functional Diversity For BinARY Distances}

\section{Attribute diversity}

For an individual assemblage, when each of the species is characterized by one or more functional traits, the pairwise distances are calculated by some distance metric, which is based on ecologically relevant species traits and characteristics relevant to ecosystem function, ecosystem services, and/or conservation; see Legendre and Legendre (2012) for various distance measures. Let $d_{i j}$ be the traitbased functional distance between the $i$ th and $j$ th species with $d_{i i}=0$ (i.e., no intraspecific variability), $d_{i j}=d_{j i} \geq 0$, $i, j=1,2, \ldots, S$. We will return to a potential extension that incorporates intraspecific variability in Conclusion and discussion. To present our theory intuitively, we here first elaborate the simplest functional diversity, where interspecific pairwise distance is a binary variable with two possible values: (1) $d_{i j}=0$ (two species are functionally identical) means that the two species are in the same 
functional group and (2) $d_{i j}=k>0$ (two species are functionally different with a constant distance $k$ ) means the two species are in different functional groups. Our approach to functional diversity is to evaluate the effective number of equally distinct functional groups. In later sections, the framework will be generalized to cases in which the degree of functional difference between any two species is reflected by a general distance between them, including continuous distance measures (e.g., Euclidean distance) or mixed-variables Gower distance. In our later generalization, the effective distance between any two species-equivalents in our simple reference assemblage is binary ( 0 or a specified positive threshold level).

Our framework is based on the attribute diversity (a generalization of Hill numbers) developed by Chao et al. (2014a). The generalization from Hill numbers to attribute diversity opens two new approaches to diversity analysis: (1) attribute diversity can be applied to any measure of species abundances, and (2) a specific attribute contribution can be defined for each species. We introduce these two ideas below, starting with the special case when pairs of species are either functionally identical or functionally different, i.e., the functional distances are binary (either 0 or $k$ ), $k>0$.

1) The measure $a_{i}$, the general abundance measure associated with species $i$, where $i=1,2, \ldots, S$. With taxonomic diversity (ordinary Hill numbers), species abundance (in the general sense) takes the form of raw measures of counts of individuals, biomass, spatial coverage, etc. for each species. For attribute diversity, we can extend species abundance to any general abundance measure associated with species $i$, called $a_{i}$. This measure is allowed to depend on the abundances of other species and may actually be greater than the abundance of that species $i$. In the simplest case of functional diversity with binary distances as described above, since two or more species may be functionally identical to one another, we expand the set of individuals belonging to species $i$ to a functionally identical set of species $i$ : the set includes all individuals, regardless of species, that have trait values identical to species $i$. The general abundance measure associated with species $i$ is defined as the abundance of the functionally identical set of species $i$. The abundance of such a functionally identical set of a particular species thus depends on the abundances of other species in the assemblage and may actually be greater than the abundance of that species $i$. For example, in the special case of binary distances, if species $j$ and $m$ are functionally identical and each is functionally different from every other species, we have $a_{j}=a_{m}=n_{j}+n_{m}$. In the special case where $n_{j}=n_{m}$, the general abundance of species $j$ is doubled; each individual of species $j$ can be regarded as being counted "twice" as the two species are functionally identical. A similar interpretation applies to species $m$.
2) The measure $v_{i}$, the attribute contribution of species $i$, where $i=1,2, \ldots, S$. We then disentangle and quantify the contribution of each species in its functionally identical set. Define the attribute contribution of species $i$ as the proportional contribution of this species to the abundance of its functionally identical set, i.e., $v_{i}=n_{i} / a_{i}$. Intuitively, because the general abundance of species $i$ is expanded to $a_{i}$, we can only have a proportion $n_{i} / a_{i}$ of its functionally identical set such that species $i$ contributes $\left(n_{i} / a_{i}\right) \times a_{i}=n_{i}$ individuals; in this proportion of functionally identical set, each individual of species $i$ is counted only once. Returning to the example for species $j$ and $m$ described in (1) in the special case where $n_{j}=n_{m}$, we have $v_{j}=n_{j} /\left(n_{j}+n_{m}\right)=1 / 2$ and $v_{m}=n_{m} /$ $\left(n_{j}+n_{m}\right)=1 / 2$. That is, since the general abundance of species $j$ would be doubled, only one-half of the functionally identical set is actually contributed by species $j$. An analogous attribute contribution applies to species $m$.

We consider three examples in the simplest binary-distances case to provide an intuitive understanding of the attribute contribution of a species in our framework.

Example 1 (binary distances).-An extreme case in which each species is equally different from every other species with a constant distance $k$. That is, the diagonal elements in the distance matrix are all 0 whereas all nondiagonal elements are a constant $k>0$. For this special case, the functionally identical set of each species comprises the individuals of only that species (i.e., $a_{i}=n_{i}$ for all $i$ ). There are no shared individuals among any two or more functionally identical sets. The attribute contribution for each species is simply unity, $v_{i}=1$ for all $i$, meaning that each species forms one functional group or contributes one functional group to the entire assemblage. Thus, this is equivalent to the framework of taxonomic diversity, i.e., there are $S$ functional groups with abundances $\left\{n_{1}, n_{2}, \ldots, n_{S}\right\}$, and thus, the effective number of functional groups reduces to Hill numbers.

Example 2 (binary distances).-The opposite extreme case is when all species are functionally identical. That is, all elements of the distance matrix are 0 . In this special case, the functionally identical set of species $i, i=1$, $2, \ldots, S$, includes all individuals in the assemblage, regardless of species, and the abundance of this single group is $n_{+}$(the total abundance of the assemblage). That is, $a_{i}=n_{+}$for $i=1,2, \ldots, S$. Each species no longer forms its own functional group, but instead contributes a proportion $p_{i}$ (species relative abundance) to the single functional group, i.e., $v_{i}=p_{i}$ for any species $i$. As will be elaborated below, our approach to functional diversity is to assess the effective number of functional groups in an assemblage in which there are $S$ sub-assemblages: the $i$ th sub-assemblage consists of $p_{i}\left(=v_{i}\right.$ in this case) functional groups (contributed by species $i$ ), each 
with group abundance $n_{+}, i=1,2, \ldots, S$. Therefore, we need to extend Hill numbers (for which each species contributes to a single functional group) to attribute diversity for which the number of functional groups contributed by each species is a proportion.

Example 3 (binary distances). - A case intermediate between the two extreme cases in Example 1 (binary distances) and Example 2 (binary distances). Suppose four equally common species are divided into two sets: $\{1,2\}$ and $\{3,4\}$. Assume that the two species within each group are functionally identical to each other and any two species from different groups are functionally different. There are two obvious functional groups: species 1 and 2 jointly form one functional group, and species 3 and 4 jointly form the other functional group. In other words, the functionally identical set of species 1 and the set of species 2 coincide and thus $a_{1}=a_{2}$; the functionally identical sets for species 3 and 4 also coincide and thus $a_{3}=a_{4}$. Since two functionally identical species jointly and equally contribute to a single functional group, each species no longer forms one group, but only one-half of a functional group, which accords with the attribute contribution of each species in this example. Consider a more general case in which the four species have unequal raw abundances $\left\{n_{1}, n_{2}, n_{3}, n_{4}\right\}$. Then, the abundances of the two functional groups become $a_{1}=a_{2}=n_{1}+n_{2}$ and $a_{3}=a_{4}=$ $n_{3}+n_{4}$, and the attribute contributions for the four species are, respectively, $v_{1}=n_{1} /\left(n_{1}+n_{2}\right), v_{2}=n_{2} /$ $\left(n_{1}+n_{2}\right), v_{3}=n_{3} /\left(n_{3}+n_{4}\right)$ and $v_{4}=n_{4} /\left(n_{3}+n_{4}\right)$. Our approach to functional diversity is to assess the effective number of functional groups for an assemblage in which there are four sub-assemblages: the first sub-assemblage consists of $n_{1} /\left(n_{1}+n_{2}\right)$ functional groups (contributed by species 1), each with group abundance $n_{1}+n_{2}$; the second sub-assemblage consists of $n_{2} /\left(n_{1}+n_{2}\right)$ functional groups (contributed by species 2), each with group abundance $n_{1}+n_{2}$; the third sub-assemblage consists of $n_{3} /\left(n_{3}+n_{4}\right)$ functional groups (contributed by species 3 ), each with group abundance $n_{3}+n_{4}$; and the fourth sub-assemblage consists of $n_{4} /\left(n_{3}+n_{4}\right)$ functional groups (contributed by species 4$)$, each with group abundance $n_{3}+n_{4}$. When all species are equally abundant (i.e., $n_{i} \equiv n$ ), each species contributes to 0.5 functional groups.

Generally, in our framework, the attribute contribution of a species is defined as the proportional number of functional groups contributed by that species. Examples 2 and 3 reveal that the attribute contributions are proportions or fractions that are less than unity, again signifying the need to extend Hill numbers to attribute diversity, which allows us to consider each species' proportional contribution, given its being functional identical to other species.

\section{Attribute diversity formula}

In Appendix S1, we use simple examples to review the concept of attribute diversity. The attribute-diversity formula corresponding to functional diversity is summarized here for an assemblage in which there are $S$ subassemblages: the $i$ th sub-assemblage consists of $v_{i}$ functional groups, each with group abundance $a_{i}, i=1,2$, $\ldots, S$. Some special cases of $a_{i}$ and $v_{i}$ are given in the above three examples for binary distances; the general formulas for $a_{i}$ and $v_{i}$ for any type of distances (including both binary and continuous distances) will be formulated in the General framework section after the introduction of the level of threshold distinctiveness.

Given a set of general species abundances, $\left\{a_{1}, a_{2}, \ldots\right.$, $\left.a_{S}\right\}$, and the corresponding set of species attribute contributions $\left\{v_{1}, v_{2}, \ldots, v_{S}\right\}$, Chao et al. (2014a) generalized Hill number of order $q$ to the following attribute diversity $(A D)$ of order $q$ :

$$
{ }^{q} A D=\left\{\sum_{i=1}^{S} v_{i}\left(\frac{a_{i}}{\bar{V}}\right)^{q}\right\}^{1 /(1-q)},
$$

where $\bar{V}=\sum_{i=1}^{S} v_{i} a_{i}$ denotes the attribute-contributionweighted total abundances. When $q=0$, we have ${ }^{0} A D=\sum_{i=1}^{S} v_{i}$ (i.e., the total attribute contribution), which represents the maximum possible value of attribute diversity. When $q$ tends to 1 , we have

$$
{ }^{1} A D=\lim _{q \rightarrow 1}{ }^{q} A D=\exp \left(-\sum_{i=1}^{S} v_{i} \frac{a_{i}}{\bar{V}} \log \frac{a_{i}}{\bar{V}}\right) .
$$

The attribute diversity ${ }^{q} A D$ quantifies the effective total attribute contribution. For functional diversity, one unit of attribute contribution represents one virtual functional group (or species-equivalent). Thus, the measure ${ }^{q} A D$ also quantifies the effective number of functional groups and ${ }^{0} A D$ becomes the total number of functional groups.

Eq. 2a shows that the attribute diversity is a genuine Hill number of order $q$ computed from an assemblage in which there are $S$ sub-assemblages: the $i$ th sub-assemblage consists of $v_{i}$ functional groups, each with group "relative" abundance $a_{i} / \bar{V}, i=1,2, \ldots, S$. Here, the sum of the "relative" abundances among all functional groups (there are $\sum_{i=1}^{S} v_{i}$ of them), as opposed to that among all species, is 1 , as reflected by the equation $\sum_{i=1}^{S} v_{i} \times\left(a_{i} / \bar{V}\right)=1$. In Example 1 (binary distances), each species has an attribute contribution of unity (i.e., $v_{i} \equiv 1$ ) and $a_{i}=p_{i}$ (relative abundance of species $i$ ), we have $\bar{V}=n_{+}$(total abundance) and Eq. 2a reduces to the Hill number of order $q$. In the special case that all abundances are identical $\left(a_{1}=a_{2}=\ldots=\right.$ $a_{S} \equiv a$ ), the attribute diversity for all $q \geq 0$ reduces to the total number of functional groups, i.e., ${ }^{q} A D=\sum_{i=1}^{S} v_{i}$, which further reduces to species richness $(S)$ if each species has an attribute contribution of unity $\left(v_{i} \equiv 1\right)$.

The attribute diversity ${ }^{q} A D$ is independent of the units used to measure abundance because it is only a function 
of the relative abundances $a_{i} / \bar{V}$ (the units in the numerator and denominator will cancel out). The measure ${ }^{q} A D$ scales proportionally with the units used to measure the attribute contribution $\left(v_{i}\right)$. That is, if all attribute contributions are multiplied by a constant $K$, then the attribute diversity becomes $K$ times the original attribute diversity for all $q \geq 0$.

\section{Attribute-Diversity Approach to Functional Diver- Sity For General Distances}

\section{Level of threshold distinctiveness}

Given any type of functional distance matrix, a widely used functional diversity measure is Rao's quadratic entropy $Q$, which represents the mean functional distance between any two individuals randomly selected from the assemblage (hence accounting for species abundance). Thus, $Q$ is also denoted by $d_{\text {mean }}$ in this paper and can be expressed as

$$
Q=d_{\text {mean }}=\sum_{i=1}^{S} \sum_{j=1}^{S} d_{i j} p_{i} p_{j} .
$$

Most previous species-equivalent methods are based on some transformation of $Q$ and a generalized version of $Q$; see Comparison with some related species-equivalent measures for details. As pointed out in Introduction, such a conventional approach has pitfalls that are prevented by introducing a new parameter $\tau$, which specifies the level of threshold distinctiveness between any two species. For a given level $\tau>0$ that determines the threshold distance, any two species with $d_{i j} \geq \tau$ are regarded as functionally equally distinct at the threshold level; for $d_{i j}<\tau$ some premises are needed.

To implement this threshold in our derivation, all distances in the distance matrix are first truncated at the level $\tau$ to obtain the following truncated distance matrix:

$$
\Delta(\tau) \equiv\left[d_{i j}(\tau)\right]=\left[\min \left(d_{i j}, \tau\right)\right] .
$$

When there are multiple assemblages, for a given value of $\tau$, the above computation could be carried out only once for the global functional distance matrix of all species in the pooled assemblage. Alternatively, we could also compute the truncated matrix for each assemblage and then pool them based on species identity. It is important to note that both ways lead to the same truncated matrix because our threshold level is fixed across all assemblages.

\section{Three basic premises}

We first explain how (virtual) functional groups are determined under some premises for a specified threshold distinctiveness level $\tau$. In later subsections, we apply attribute diversity to quantify the effective number of equally distinct functional groups. For clarity, our following formulation is based on species raw abundances (the number of individuals) $\left\{n_{1}, n_{2}, \ldots, n_{S}\right\}$; a parallel derivation holds when raw abundances are replaced by species relative abundances, biomass, cover, etc. To form virtual functional groups in our framework, we have the following three basic premises for any pair of species $(i, j)$ :

Premise A.-If $d_{i j}=0$, then $d_{i j}(\tau)=0$ for any level $\tau>0$. The two species are always treated as belonging to the same functional group.

Premise B.-If $d_{i j} \geq \tau$, then $d_{i j}(\tau)=\tau$ and species $i$ and species $j$ are regarded as functionally equally distinct at the given threshold level $\tau$ and are always treated as belonging to different functional groups with an effective distance $\tau$.

Premise C.-For an intermediate situation where $0<d_{i j}<\tau$, we have $d_{i j}(\tau)=d_{i j}$. In this case, we assume that only a proportion of the individuals of species $i$ are functionally equally distinct from species $j$ at the threshold distinctiveness level $\tau$ (and thus are in different functional groups with an effective distance $\tau$ ), whereas the other individuals of species $i$ are functionally indistinct from species $j$ (i.e., in the same functional group with an effective distance 0 ). Also, the same proportion of individuals of species $j$ are functionally equally distinct from species $i$, whereas the other individuals of species $j$ are functionally indistinct from species $i$. It is thus necessary to define a monotonically increasing function $f\left(d_{i j}(\tau)\right)$ to compute the proportion of individuals of one species that are functionally equally distinct from the other species at a given level $\tau$. This function must satisfy the following two boundary conditions: $f(0)=0$ (proportion is 0 if $d_{i j}=0$, as in Premise $A$ ) and $f(\tau)=1$ (proportion is unity if $d_{i j} \geq \tau$, as in Premise B). For a specified function $f$, we assume that proportionally there are $f\left(d_{i j}(\tau)\right) \times n_{j}$ individuals of species $j$ functionally equally distinct from species $i$ with an effective distance $\tau$, whereas the other $\left[1-f\left(d_{i j}(\tau)\right)\right] \times n_{j}$ individuals are functionally indistinct from species $i$ with an effective distance 0 . A similar computation could be made for individuals of species $i$.

There are many choices for the monotonically increasing function $f$ with $f(0)=0$ and $f(\tau)=1$. Our following derivation is valid for any monotonically increasing function $f$ satisfying the two boundary conditions at 0 and $\tau$ unless otherwise stated. For illustration purposes, our examples and real data analysis are based on the simple linear function $f\left(d_{i j}(\tau)\right)=d_{i j}(\tau) / \tau$.

\section{General framework}

To formulate our proposed functional diversity in terms of attribute diversity (Eq. 2a) for any type of species-pairwise distance matrix $\left[d_{i j}\right]$ under a specified level of threshold distinctiveness $\tau$, we need to determine a general abundance measure for each species $i$ (i.e., $a_{i}, i=1,2, \ldots$, 
$S$, in Eq. 2a and for its corresponding attribute contribution (i.e., $v_{i}, i=1,2, \ldots, S$, in Eq. $2 \mathrm{a}$, as described below and illustrated in later examples.

1) The measure $a_{i}(\tau)$, the general abundance measure associated with species $i$ for a specified threshold level $\tau$, where $i=1,2, \ldots, S$. First, we need to extend the functionally identical set (actual trait-based distance $=0$ ) of a species, introduced in the section for binary distances, to make a functionally indistinct (effective distance $=0$ ) set at the threshold distinctiveness $\tau$. For any specified level $\tau$, define the functionally indistinct set (or $f$-indistinct set) of species $i$ as the collection of all individuals in the assemblage that are $f$-indistinct from species $i, i=1$, $2, \ldots, S$. Given the above three premises and any function $f$ in $[0,1]$ with $f(0)=0$ and $f(\tau)=1$, this $f$-indistinct set $i$ includes all individuals of species $i$ and a fraction $\left[1-f\left(d_{i j}(\tau)\right)\right]$ of the individuals of any other species $j$. The abundance of this set is denoted as $a_{i}(\tau)$ to emphasize that it is a function of the level $\tau$, and is thus expressed as a function of all species' raw abundances and species-pairwise distances

$$
a_{i}(\tau)=n_{i}+\sum_{j \neq i}\left[1-f\left(d_{i j}(\tau)\right)\right] n_{j}=\sum_{j=1}^{S}\left[1-f\left(d_{i j}(\tau)\right)\right] n_{j} .
$$

2) The measure $v_{i}(\tau)$, the attribute contribution of species $i$ for a threshold level $\tau$, where $i=1,2, \ldots, S$. If species $i$ is functionally equally distinct from all other species at the threshold level $\tau$, i.e., the trait-based distance between species $i$ and any other species is at least $\tau$, then it follows from Eq. $3 \mathrm{c}$ that we have $a_{i}(\tau)=n_{i}$, and no individual of species $i$ is shared in any other $f$-indistinct sets. This species with $n_{i}$ individuals thus contributes a single virtual functional group. On the other hand, if there exists at least one species $j$ such that $d_{i j}<\tau$, then the $f$-indistinct set of species $i$ includes some individuals of other species so that $a_{i}(\tau)>n_{i}$. In this case, species $i$ no longer contributes to a single functional group, but a proportion $n_{i} / a_{i}(\tau)$ of a functional group. We thus define this proportion as the attribute contribution of species $i$ (i.e., $v_{i}$ in Eq. 2a) and denote it as $v_{i}(\tau)$ for a given threshold level $\tau$

$$
\begin{aligned}
v_{i}(\tau) & =n_{i} / a_{i}(\tau)=n_{i} / \sum_{j=1}^{S}\left[1-f\left(d_{i j}(\tau)\right)\right] n_{j} \\
& =p_{i} / \sum_{j=1}^{S}\left[1-f\left(d_{i j}(\tau)\right)\right] p_{j} .
\end{aligned}
$$

Here $p_{i}=n_{i} / n_{+}, i=1,2, \ldots, S$, denotes species relative abundances. The attribute contribution $v_{i}(\tau)$ is defined as the abundance of species $i$ divided by the combined abundance (i.e., $a_{i}(\tau)$ ) of all functionally indistinct individuals from species $i$. The attribute contribution of species $i$ represents the proportional number of functional groups contributed by species $i, i=1,2, \ldots, S$. Since $a_{i}(\tau)$ is between $n_{i}$ and $n_{+}$, i.e., $n_{i} \leq a_{i}(\tau) \leq n_{+}$, it follows that $p_{i} \leq v_{i}(\tau) \leq 1$.
The maximum attribute contribution of unity is attained in the extreme case when functional diversity reduces to classic taxonomic diversity (Example 1 (general distances)), whereas the minimum attribute contribution is attained in the opposite extreme case when all species are functionally identical (Example 2 (general distances)).

We now show that the three simple examples for binary distances (i.e., distance is either 0 or $k$ ) are special cases of our general framework if the threshold level is specified to be $k$. We also apply Eqs. $3 \mathrm{c}$ and $3 \mathrm{~d}$ to rigorously obtain those intuitive results given earlier.

Example 1 (general distances).- - If $d_{i j} \geq \tau$ for all $i \neq j$, then Premise B implies that each species is equally distinct from every other species at a constant effective distance $\tau$. This reduces to the binary-distances case (effective distance is either 0 or $\tau$ ). Eqs. $3 \mathrm{c}$ and $3 \mathrm{~d}$ lead to $a_{i}(\tau)=n_{i}$ and $v_{i}(\tau)=1$ for any function $f$. As discussed in Example 1 (binary distances), there are $S$ functional groups with abundances $\left\{n_{1}, n_{2}, \ldots, n_{S}\right\}$, which is equivalent to the framework of taxonomic diversity. Unlike taxonomic diversity, however, our functional diversity measure also incorporates the important information that the distance between any two functional groups or species-equivalents is at least $\tau$.

Example 2 (general distances).- In the opposite extreme case that $d_{i j}=0$ for all species pairs, Premise A implies that all species are in one functional group. It follows from Eqs. 3c and $3 \mathrm{~d}$ that $a_{1}(\tau)=\ldots=a_{S}(\tau)=$ $\sum_{i=1}^{S} n_{i} \equiv n_{+}$, and $v_{i}(\tau)=n_{i} / n_{+}=p_{i}$ for any level $\tau>0$. The $S$ functionally indistinct sets coincide. Each species contributes a proportion $p_{i}$ (species relative abundance) to the single functional group. The functional diversity in this case, as will be shown later, is always unity for any level $\tau>0$ when all species are functionally identical.

Example 3 (General distances).-Consider the case that the four species in the assemblage described earlier have unequal raw abundances $\left\{n_{1}, n_{2}, n_{3}, n_{4}\right\}$. Assume that two species within any group have distance 0 whereas two species from different groups have trait-based distance $\geq \tau$. This reduces to the binary-distances case (effective distance is either 0 or $\tau$ ). Based on Eq. 3c, we have $a_{1}(\tau)=a_{2}(\tau)=n_{1}+n_{2}$ and $a_{3}(\tau)=a_{4}(\tau)=n_{3}+n_{4}$, so the $f$-indistinct sets for species 1 and 2 coincide each with abundance $n_{1}+n_{2}$. The contributions from the two species based on Eq. $3 \mathrm{~d}$ are, respectively, $v_{1}(\tau)=n_{1} / a_{1}(\tau)=$ $n_{1} /\left(n_{1}+n_{2}\right)$ and $v_{2}(\tau)=n_{2} / a_{2}(\tau)=n_{2} /\left(n_{1}+n_{2}\right)$. Analogously, the $f$-indistinct sets for species 3 and 4 coincide each with abundance $n_{3}+n_{4}$; the contributions from the two species are, respectively, $v_{3}(\tau)=n_{3} / a_{3}(\tau)=$ $n_{3} /\left(n_{3}+n_{4}\right)$ and $v_{4}(\tau)=n_{4} / a_{4}(\tau)=n_{4} /\left(n_{3}+n_{4}\right)$.

\section{Functional diversity formulas}

Given a species-pairwise distance matrix $\left[d_{i j}\right]$ and a specified threshold distinctiveness level $\tau>0$, we first 
form the $f$-indistinct set of each of the $S$ species in the assemblage. Their abundances, $a_{i}(\tau), i=1,2, \ldots, S$, are formulated in Eq. 3c. If all these sets are disjoint (no shared individuals among any two or more $f$-indistinct sets), then our functional diversity reduces to classic taxonomic diversity, for which each species contributes one functional group. Typically, two or more of these $f$-indistinct sets overlap to some extent. We then quantify the contribution of each species via the proportion, $v_{i}(\tau)=n_{i} / a_{i}(\tau), \quad i=1,2, \ldots, \quad S$; see Eq. 3d. Our approach to functional diversity is to assess the effective number of equally distinct functional groups in an assemblage in which there are $S$ sub-assemblages: the $i$ th sub-assemblage consists of a proportional number $v_{i}(\tau)$ of functional groups, each with group abundance $a_{i}(\tau)$, $i=1,2, \ldots, S$. This fits the framework of attribute diversity (Eq. 2a). Substituting $a_{i}, v_{i}$, and $\bar{V}$ in Eq. 2a by $a_{i}(\tau), v_{i}(\tau)$ and $\bar{V}(\tau)=\sum_{i=1}^{S} v_{i}(\tau) a_{i}(\tau)=\sum_{i=1}^{S} n_{i}=n_{+}$, $i=1,2, \ldots, S$, we obtain the functional diversity of order $q$ at level $\tau>0$ as the attribute diversity of order $q$

$$
\begin{gathered}
{ }^{q} F D(\Delta(\tau))=\left(\sum_{i=1}^{S} v_{i}(\tau)\left(\frac{a_{i}(\tau)}{n_{+}}\right)^{q}\right)^{1 /(1-q)} \\
=\left(\sum_{i=1}^{S} p_{i}\left(\sum_{j=1}^{S}\left[1-f\left(d_{i j}(\tau)\right)\right] p_{j}\right)^{q-1}\right)^{1 /(1-q)} .
\end{gathered}
$$

Eq. 4a also reveals that the functional diversity of order $q$ is simply the Hill number of order $q$ for an assemblage in which there are $S$ sub-assemblages with the $i$ th sub-assemblage consisting of $v_{i}(\tau)=n_{i} / a_{i}(\tau)$ functional groups, each with group "relative" abundance $a_{i}(\tau) / n_{+}, i=1,2, \ldots, S$. Here, the sum of these "relative" abundances over all functional groups is unity, as reflected by the equation $\sum_{i=1}^{S} v_{i}(\tau)\left[a_{i}(\tau) / n_{+}\right]=1$.

Although our framework is formulated in terms of species raw abundances, the proposed functional diversity is eventually only a function of species relative abundances (Eq. 4b). The functional diversity ${ }^{q} F D(\Delta(\tau)$ ) quantifies the effective number of equally distinct functional groups (or species) at the threshold distinctiveness of level $\tau$, equivalently, the distance between any two species-equivalents is at least $\tau$ in the reference assemblage. The sense of "effective" is thus the following: if ${ }^{q} F D(\Delta(\tau))=x$, then the functional diversity of the actual assemblage is the same as a reference assemblage consisting of $x$ equally abundant and functionally equally distinct species with all pairwise distances $\geq \tau$ for any different-species pairs, and distance 0 for any samespecies pairs; see Example 1 (general distances).

\section{Properties of our functional diversity}

The proposed functional diversity possesses the following properties:
Range. - The measure ${ }^{q} F D(\Delta(\tau))$ takes a value between 1 and $S$. When all species are functionally identical (i.e., all pairwise functional distances are equal to 0 , as in Example 2 (general distances), ${ }^{q} F D(\Delta(\tau))$ attains its minimal value of unity for all $q \geq 0$, implying there is only one species-equivalent in the reference assemblage. When only one species dominates the assemblage (i.e., $p_{1} \rightarrow 1$ and $\left.\sum_{j>1} p_{j} \rightarrow 0\right),{ }^{q} F D(\Delta(\tau))$ also approaches unity for all $q>0$, regardless of the functional distance between any two species. The measure ${ }^{q} F D(\Delta(\tau))$ attains the maximum value of $S$ for all $q \geq 0$ when all species are equally abundant and all trait-based distances for different-species pairs are at least $\tau$ so that all species are functionally equally distinct at the level $\tau$ (see Example 1 (general distances)).

Two-group assemblage.-Suppose all species in an assemblage are equally abundant, and that these species are evenly divided into two groups in such a way that all species within a group have identical trait values, and species from different groups have a distance $b$. Then for any $\tau, 0<\tau \leq b$, it is easy to verify that ${ }^{q} F D(\Delta(\tau))=2$ for all $q \geq 0$, correctly implying that there are two functional groups (see Example 3 (general distances)). When $f\left(d_{i j}(\tau)\right)=d_{i j}(\tau) / \tau$ and $\tau>b$ we have ${ }^{q} F D(\Delta(\tau))=$ $1 /[0.5+0.5(1-b / \tau)]$ for all $q \geq 0$, correctly implying that there are two functional groups when $\tau$ tends to $b$, and there is only one group when $\tau$ tends to infinity. The same results emerge for any other function $f$ with $f$ $(0)=0$ and $f(\tau)=1$.

Order zero.-For $q=0$, we have

$$
\begin{aligned}
{ }^{0} F D(\Delta(\tau)) & =\sum_{i=1}^{S} v_{i}(\tau)=\sum_{i=1}^{S} \frac{n_{i}}{a_{i}(\tau)} \\
& =\sum_{i=1}^{S} \frac{p_{i}}{\sum_{j=1}^{S}\left[1-f\left(d_{i j}(\tau)\right)\right] p_{j}}
\end{aligned}
$$

which is the functional richness or the total number of functional groups at the threshold level $\tau$. This differs from the two currently used measures of functional richness, namely FRic and FAD, which, respectively, refer to the convex hull volume and the sum of pairwise distances (Mouchet et al. 2010). Unlike these two measures, our functional diversity of order zero is allowed to be a function of species abundances, because here each attribute contribution is designed to account for species abundances (see Eq. 3d) and, thus, the total attribute contribution depends on species abundances. When only species presence/absence data are available, we can assume that all species are equally abundant in Eq. 4c. This leads to a functional richness measure ${ }^{0} F D(\Delta(\tau))=\sum_{i=1}^{S}$ $\left(\sum_{j=1}^{S}\left[1-f\left(d_{i j}(\tau)\right)\right]\right)^{-1}$, which does not account for species abundances. 
Order one.-For $q=1$, we have

$$
\begin{aligned}
{ }^{1} F D(\Delta(\tau)) & =\lim _{q \rightarrow 1}{ }^{q} F D(\Delta(\tau)) \\
& =\exp \left(-\sum_{i=1}^{S} v_{i}(\tau) \frac{a_{i}(\tau)}{n_{+}} \log \left(\frac{a_{i}(\tau)}{n_{+}}\right)\right) \\
& =\exp \left(-\sum_{i=1}^{S} p_{i} \log \left(\sum_{j=1}^{S}\left[1-f\left(d_{i j}(\tau)\right] p_{j}\right)\right) .\right.
\end{aligned}
$$

The above measure represents a generalization of the Shannon-like measure proposed in Ricotta and Szeidl (2006).

Order two. - For $q=2$, we have

$$
{ }^{2} F D(\Delta(\tau))=\frac{1}{1-\sum_{i=1}^{S} \sum_{j=1}^{S} p_{i} f\left[d_{i j}(\tau)\right] p_{j}} .
$$

In the special simple linear function, $f\left(d_{i j}(\tau)\right)=d_{i j}(\tau) / \tau$, the above reduces to

$$
{ }^{2} F D(\Delta(\tau))=\frac{1}{1-\sum_{i=1}^{S} \sum_{j=1}^{S} p_{i}\left[d_{i j}(\tau) / \tau\right] p_{j}} \equiv \frac{1}{1-Q(\Delta(\tau)) / \tau},
$$

where $Q(\Delta(\tau))$ denotes the quadratic entropy based on the distance matrix $\Delta(\tau)$, i.e.,

$$
Q(\Delta(\tau))=\sum_{i=1}^{S} \sum_{j=1}^{S}\left[d_{i j}(\tau)\right] p_{i} p_{j}
$$

The species-equivalent formula for quadratic entropy and its corresponding replication principle have been extensively discussed in the literature (e.g., Ricotta and Szeidl 2009, Ricotta and Acosta 2014, Gregorius and Kosman 2017). Our formula in Eq. 4e in terms of a given threshold level provides a novel modification to previous formulas and resolves some limitations associated with existing formulas; see Comparisons with some related species-equivalent measures for comparisons.

Replication principle. - For any given threshold distinctiveness level $\tau>0$, the functional diversity ${ }^{q} F D(\Delta(\tau))$ satisfies a replication principle for $N$ equally large (i.e., equal total-abundance) and equally diverse assemblages. Let $\left[d_{i j}^{(k)}\right]$ denote the species-pairwise distance matrix of the $k$ th assemblage and $\Delta_{k}(\tau) \equiv\left[\min \left(d_{i j}^{(k)}, \tau\right)\right]$ denote the corresponding truncated matrix for the level of threshold distinctiveness $\tau$. For any $\tau>0$, assume all assemblages have the same functional diversity, i.e., ${ }^{q} F D$ $\left(\Delta_{1}(\tau)\right)={ }^{q} F D\left(\Delta_{2}(\tau)\right)=\ldots={ }^{q} F D\left(\Delta_{N}(\tau)\right)$. When there are no shared species among the $N$ assemblages, and the distance of any two species from different assemblages is at least $\tau$, then the functional diversity of the pooled assemblage is $N$ times the diversity of each assemblage. See Appendix S1 for a proof.

When a functional diversity measure is based only on species-pairwise distances (without considering species abundances), Ricotta (2005) listed three properties that a meaningful measure should satisfy: "twinning property," "distance monotonicity," and "set monotonicity." When species abundances are also considered, we prove in Appendix S1 that our measure ${ }^{q} F D(\Delta(\tau))$ satisfies similar properties under some modifications.

Twinning.-Suppose some individuals of a species are replaced by individuals of a new species with the same traits ("twin" species). Then, the functional diversity ${ }^{q} F D(\Delta(\tau))$ does not change for all $q \geq 0$ and $\tau>0$.

Monotonicity with functional distance.-The functional diversity ${ }^{q} F D(\Delta(\tau))$ is never decreased by an increase in the trait-based distances between species. Specifically, suppose one non-diagonal element (say, $d_{k m}$ ) of a species-pairwise distance matrix increases from $d_{k m}$ to $d_{k m}+c$, where $c>0$. Then, the functional diversity ${ }^{q} F D$ $(\Delta(\tau))$ does not decrease. Moreover, when $\tau>d_{k m}$, functional diversity ${ }^{q} F D(\Delta(\tau))$ always increases for all $q \geq 0$.

Monotonicity with the addition of functionally distant species. - Like species richness, our functional richness satisfies a monotonicity property. The functional diversity of order zero, ${ }^{0} F D(\Delta(\tau))$, always increases by one when a species added to an assemblage is functionally equally distinct from all species in the original assemblage at the threshold level $\tau$. However, one additional assumption (i.e., the added species is the rarest one in the augmented assemblage) is needed so that the functional diversity ${ }^{q} F D(\Delta(\tau))$ for $q>0$ always increases; see Appendix S1 for proofs and examples.

\section{Comparisons with Some Related Species-Equivalent Measures}

As presented in Introduction, Ricotta and Szeidl (2006) proposed a class of distance-based Tsallis-type entropy based on a standardized distance matrix $\left(d_{i j}\right)$ with $0 \leq d_{i j} \leq 1$ (this is equivalent to normalizing the distance by means of dividing each pairwise functional distance by the maximum distance between two species)

$$
{ }^{q} H_{R S}=\frac{1}{q-1}\left(1-\sum_{i=1}^{S} p_{i}\left(1-\sum_{j \neq i} d_{i j} p_{j}\right)^{q-1}\right)
$$

where the sub-index $R S$ refers to Ricotta and Szeidl. In the special case of $q=2$, this measure reduces to $Q$. When all distances are between 0 and 1, Ricotta and Szeidl (2009) and de Bello et al. (2010) proposed transforming $Q$ to the following species-equivalent formula: 


$$
Q_{e}=\frac{1}{1-Q},
$$

where the sub-index $e$ refers to "effective." It is interpreted as the number of equally abundant and maximally distinct species with a constant distance of 1 between any two species. For distances that do not have an upper bound, the above species-equivalent formula was modified to

$$
Q_{e}\left(d_{\max }\right)=\frac{1}{1-Q / d_{\max }},
$$

where $d_{\max }$ denotes the maximum of species-pairwise distances in the observed data. Similar to Eq. 5b, this number can be interpreted as being in terms of maximal distinct species, but with a constant distance of $d_{\max }$ between any two species.

Leinster and Cobbold (2012) proposed a class of similarity-based species-equivalent formulas. A key component in their approach is a transformation converting distance to similarity. Assume a similarity matrix $\left[Z_{i j}\right]$ can be defined, where $Z_{i j}$ denotes the similarity between species $i$ and species $j, 0 \leq Z_{i j} \leq 1$. Their species-equivalent formula is expressed as

$$
{ }^{q} D_{L C}=\left(\sum_{i=1}^{S} p_{i}\left(\sum_{j=i}^{S} Z_{i j} p_{j}\right)^{q-1}\right)^{1 /(1-q)},
$$

where the sub-index $L C$ refers to Leinster and Cobbold. They interpreted the above formula as the effective number of totally distinct species-equivalents (i.e., the similarity for any two different species-equivalents in the reference assemblage is 0 ).

In the special cases that $Z_{i j}=1-d_{i j}$ (when the distance metric has an upper bound of unity) and $Z_{i j}=1-d_{i j} / d_{\max }$ (when each distance is divided by the maximum distance), Eq. 5 d represents an extension of Eqs. $5 \mathrm{~b}$ and $5 \mathrm{c}$ to any diversity order $q \geq 0$. Comparing these three conventional formulas given in Eqs. $5 b-5 d$ with our measures in $4 \mathrm{~b}$ and $4 \mathrm{e}$, we see the conventional species-equivalent numbers are special cases of our functional diversity when a simple linear function, $f$ $\left(d_{i j}(\tau)\right)=d_{i j}(\tau) / \tau$, is applied and the threshold level is set to be an upper bound $\tau=1$ (for Eqs. $5 \mathrm{~b}$ and $5 \mathrm{~d}$ with $Z_{i j}=1-d_{i j}$ ) or a maximum level $\tau=d_{\max }$ (for Eqs. $5 \mathrm{c}$ and $5 \mathrm{~d}$ with $Z_{i j}=1-d_{i j} / d_{\max }$ ).

When distance metrics are not bounded, Leinster and Cobbold advocated using the exponential transformation, $Z_{i j}=\exp \left(-u d_{i j}\right), u>0$. However, as pointed out by Botta-Dukát (2018), the transformed similarity cannot attain 0 under their proposed transformation $\exp \left(-u d_{i j}\right)$ for finite distances. Thus, there is no "attainable" simple reference assemblage for the "effective" sense. Leinster and Cobbold (2012:487) discussed an alternative linear truncated transformation and Botta-Dukát (2018) later proposed a truncated exponential function. Using a truncated function is equivalent to setting a threshold of distinctiveness so that the reference assemblage is attainable.

By implementing a threshold distinctiveness level, our distance-based approach avoids the transformation to similarity and can be applied to any kind of species-pairwise distance matrix (bounded or unbounded) and any general function $f$. In addition, our reference assemblage (in which two species-equivalents are either functionally indistinct or functionally equally distinct at the threshold distinctiveness level $\tau$ ) is attainable, and thus, the interpretation of "effective" is more transparent. Most importantly, as we show in Functional diversity decomposition, our attribute-diversity approach leads to a diversity decomposition with gamma never less than alpha, and the beta component can be used to construct various functional (dis)similarity measures.

As discussed in Introduction, previous authors have indicated in real data analyses that formulas $(5 b-5 d)$ typically yield low diversity values in functional diversity studies especially when there are many species, and species-pairwise distance is computed using Gower distance. From our perspective, the weak sensitivity of these conventional measures to distance matrices and species abundances is mainly due to the threshold distinctiveness level being set to be a maximum or the upper bound of the distance metric. With a high threshold level, nearly all functionally indistinct sets are relatively large and tend to include most individuals in the assemblage unless some species pairs are maximally distinct. Intuitively, imagine that all species are placed in a functional space with specified pairwise distances; if we use a very high threshold distance to cluster them into different functional groups, then nearly all species are in the same group unless some species-pairwise distances attain that high threshold level. Indeed, in real study cases, there are many more species than functional traits used to describe them (usually more than 30 species and less than 10 traits), while representing $M$ maximally distinct species at the upper bound of the Euclidean space requires at least $M-1$ axes (e.g., equilateral triangle in two dimensions for three species, regular tetrahedron in three dimensions for four species). Hence, only very few species pairs can have distances close to the maximal distance; even if those species are the most abundant, the conventional formulas ( $5 \mathrm{~b}-5 \mathrm{~d}$ ) will necessarily yield low values.

Ricotta and Acosta (2014) relaxed the stringent requirement of "maximal distinction" to allow for species to be "partially distinct" so that equivalent species are equally distinct from each other with a constant effective distance of $d^{\prime}$ in their reference assemblage. Their $d^{\prime}$ by definition is different from the threshold distinctiveness level $\tau$. The difference lies in the fact that we calculate quadratic entropy, $Q(\Delta(\tau))$ (Eq. 4e), based on a truncated matrix whereas theirs is based on the original distance matrix. In our definition, equivalent species are equally distinct from each other with species-pairwise distance at 
least $\tau$ for different species-pairs. Consequently, their species-equivalent formula, given below, is different from our $q=2$ formula given in Eq. $4 \mathrm{e}$.

$$
Q_{e}\left(d^{\prime}\right)=\frac{1}{1-Q / d^{\prime}} .
$$

There are some restrictions in their approach: (1) the value of $d^{\prime}$ must be greater than $Q$ otherwise a negative value or infinity is obtained, (2) the effective number can be greater than the actual species richness, and (3) diversity partitioning that decomposes gamma into alpha and beta is not available. By contrast, our species-equivalent formula in (4e) yields diversity values in $[1, S]$ and can quantify the effective number of equally distinct species where an effective distance between any two speciesequivalents can be any positive number. In addition, proper decomposition can be developed, as will be shown in Functional diversity decomposition. Note that the species-equivalent formula $5 \mathrm{e}$ cannot be linked to taxonomic diversity (Hill number of order 2) except for a special type of distances $\left(d_{i j}=1\right.$ for $\left.i \neq j\right)$, whereas our formula $4 \mathrm{e}$ reduces to taxonomic diversity for any type of distance matrix if we let $\tau$ tend to 0 . Thus, implementing a threshold distinctiveness level to the original distance matrix is essential to avoiding all the restrictions stated above. Gregorius and Kosman (2017) recently proposed two classes of dispersion measures and derived the corresponding effective numbers. Their speciesequivalent formula is similar to Eq. 5e but with $Q$ being replaced by a class of dispersion measure and $d^{\prime}$ being referred to as the level of distinction. Since their measures include Ricotta and Acosta's (2014) index as a special case, similar restrictions mentioned above for Eq. 5e also apply to their measures. For example, Gregorius and Kosman (2017) indicated that $d^{\prime}$ must be greater than the dispersion value to yield a positive finite effective number.

Chiu and Chao (2014) developed a class of functional diversity measures that quantifies the effective total functional distance; their induced species-equivalent formula quantifies the effective number of functionally equally distinct species with a constant distance of $Q$ (quadratic entropy) between any two species-equivalents. In this paper, when the level of threshold distinctiveness $\tau=d_{\text {mean }}=Q$, the proposed functional diversity quantifies the effective number of functionally equally distinct species with pairwise distances for different-species pairs at least $Q$. Consequently, Chiu and Chao's distanceequivalent perspective is not directly comparable to the current approach due to different definitions of speciesequivalents. Scheiner et al. (2017) derived several functional diversity indices under a framework of Hill numbers. As indicated by Scheiner et al. (2017), their indices are abundance-weighted measures of functional-trait diversity and thus quantify different aspects of trait space. For example, in the special case that only species-pairwise distances are considered, their measure quantifies the diversity of proportional distances and thus possesses different properties from our proposed functional diversity.

\section{A Simple Hypothetical Example}

We use a hypothetical example to illustrate our framework based on the simple linear function, $f$ $\left(d_{i j}(\tau)\right)=d_{i j}(\tau) / \tau$. Consider the following species pairwise functional distance matrix of four species $(1,2,3,4)$

$$
\left[\begin{array}{llll}
0 & 2 & 8 & 8 \\
2 & 0 & 8 & 8 \\
8 & 8 & 0 & 6 \\
8 & 8 & 6 & 0
\end{array}\right]
$$

Assume the raw abundances for the four species (1, 2, 3, 4) are $\left\{n_{i} ; i=1,2,3,4\right\}=(20,15,10,5)$ with total abundance $n_{+}=50$. The species relative abundances are $\left\{p_{i}=n_{i} / n_{+} ; i=1,2,3,4\right\}=\left(\begin{array}{llll}0.4, & 0.3, & 0.2, & 0.1\end{array}\right)$. Although our functional diversity depends only on species relative abundances, we use raw abundances for illustrative purposes. In the matrix data, the minimum distance between two different species is $d_{\min }=2$ and the maximum distance is $d_{\max }=8$. We choose for illustration that the traits of species 1 and species 2 are very similar $\left(d_{12}=2\right)$, those of species 3 and 4 are moderately similar $\left(d_{34}=6\right)$, and the maximal distance is maintained between all cross-group pairs of species for the two groups $(1,2)$ and $(3,4)\left(d_{13}=d_{14}=d_{23}=d_{24}=\right.$ $\left.d_{\max }=8\right)$. The average distance is $d_{\text {mean }}=Q=4.08$ (species-abundance-weighted average distance; see Eq. 3a). In the following, we consider several choices of level of threshold distinctiveness.

All results with illustrative figures are shown in Fig. 1. In our interpretation of effective numbers, "species (equivalent)" and "(virtual) functional group" are synonyms. For this example, our functional diversity for each level $\tau$ quantifies the effective number of functional groups (or species) for an assemblage in which there are four subassemblages: the $i$ th sub-assemblage consists of $v_{i}(\tau)=n_{i} /$ $a_{i}(\tau)$ functional groups, each with group abundance $a_{i}(\tau)$ and "relative" abundance $a_{i}(\tau) / n_{+}$. Here, the sum of group "relative" abundances among all functional groups is unity, i.e., $\sum_{i=1}^{S} v_{i}(\tau)\left[a_{i}(\tau) / n_{+}\right]=1$. In Fig. 1, for each species $i=1,2,3$, and 4 , we list its attribute contribution, group abundance, and the "relative" abundance.

1) We first consider a level of threshold distinctiveness $\tau=d_{\min }=2$. Under this level, all distances in the distance matrix are truncated at 2 to become $\Delta(2) \equiv$ $\left[d_{i j}(2)\right]=\left[\min \left(d_{i j}, 2\right)\right]$, i.e.,

$$
\Delta(2)=\left[\begin{array}{llll}
0 & 2 & 2 & 2 \\
2 & 0 & 2 & 2 \\
2 & 2 & 0 & 2 \\
2 & 2 & 2 & 0
\end{array}\right]
$$




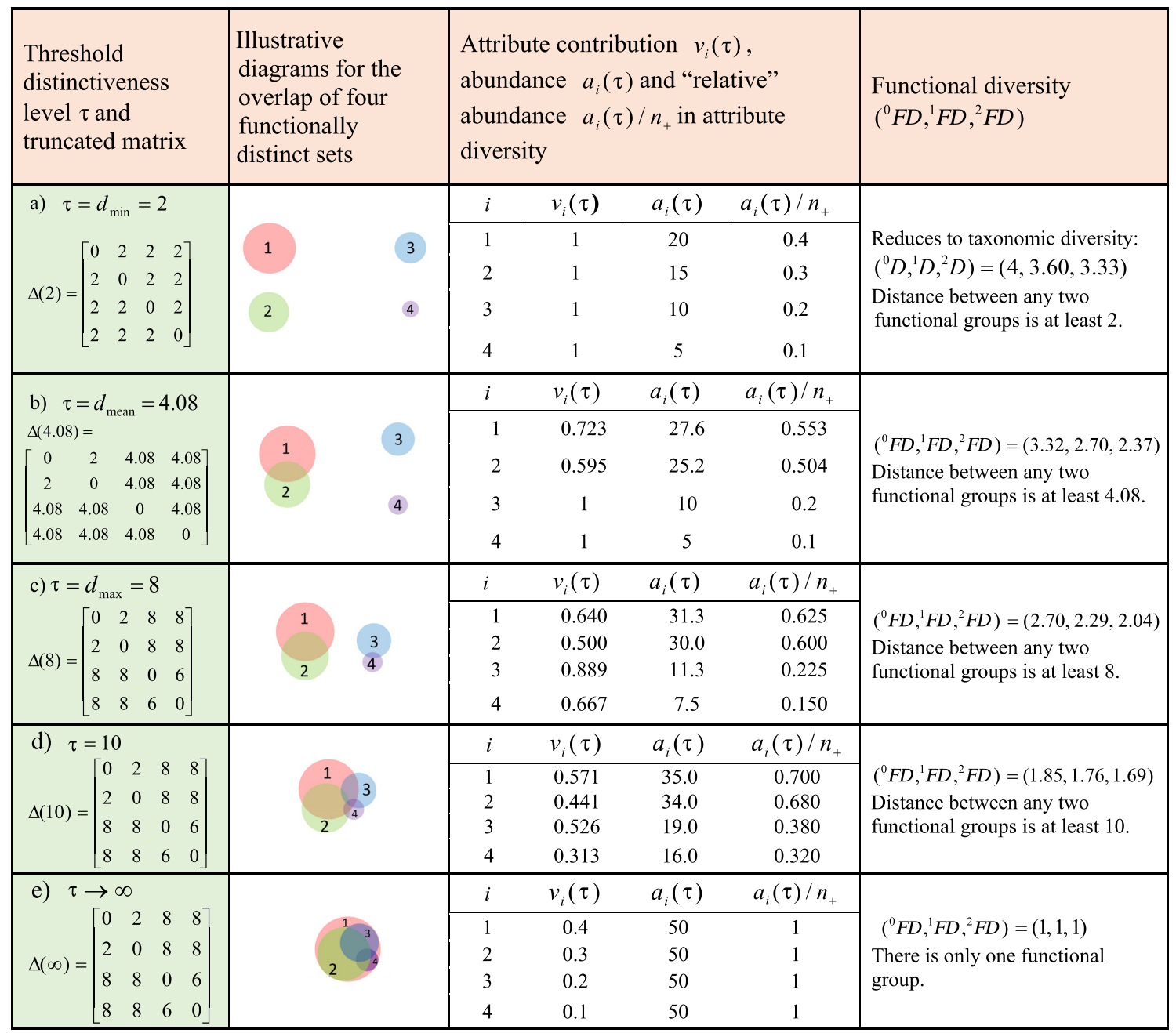

FIG. 1. Illustration of truncated matrices and the overlap of functionally indistinct sets in an assemblage of four species $(1,2,3$, 4) for varying levels of threshold distinctiveness (a-e) along with the attribute contributions and the corresponding abundances in the attribute-diversity approach to functional diversity. The number of individuals for the four species in an assemblage is $\left\{n_{i} ; i=1,2,3,4\right\}=(20,15,10,5)$ with total abundance $n_{+}=50$. The original functional distance matrix for the four species is shown in panels $\mathrm{c}, \mathrm{d}$, and e. The area of each circle represents the magnitude of species abundance. As the threshold distinctiveness level is increased, some functionally indistinct sets overlap; when the level is infinity, all functionally indistinct sets coincide. Our approach considers an assemblage that is composed of four sub-assemblages: the $i$ th sub-assemblage $(i=1,2,3$, and 4$)$ consists of $v_{i}(\tau)$ functional groups with abundance $a_{i}(\tau)$ and "relative" abundance $a_{i}(\tau) / n_{+}$, which satisfies $\sum_{i=1}^{4} v_{i}(\tau)\left[a_{i}(\tau) / n_{+}\right]=1$. The functional diversity is computed as the attribute diversity of the assemblage: ${ }^{q} F D \equiv{ }^{q} F D(\Delta(\tau))=\left(\sum_{i=1}^{S} v_{i}(\tau)\left[a_{i}(\tau) / n_{+}\right]^{q}\right)^{1 /(1-q)}$, and is interpreted as the number of equally abundant and equally distinct functional groups (or species) with pairwise distances at least $\tau$; see Eqs. $4 \mathrm{a}-4 \mathrm{e}$.

All species are thus functionally and equally distinct from each other at a threshold level $\tau=2$. As discussed in Example 1 above, we have $a_{i}(\tau)=n_{i}, i=1,2,3,4$, and the attribute contribution for each species is unity as reflected by $\left\{v_{i}(\tau)=n_{i} / a_{i}(\tau), i=1,2,3,4\right\}=\{1,1,1$, 1 \}. There are four functional groups, each with relative abundances $\left\{a_{i}(\tau) / n_{+} ; i=1,2,3,4\right\}=\{0.4,0.3,0.2$, $0.1\}$. Thus, functional diversity (the effective number of functional groups) reduces to the ordinary Hill number based on species abundances. This is true not only for $\tau=d_{\min }$ but also for any level of threshold distinctiveness $\tau \leq d_{\text {min }}$. For Hill numbers (Eqs. 1a and 1b), we have ${ }^{0} D=4,{ }^{1} D=3.60$, and ${ }^{2} D=3.33$. For $q=0,1$, and 2, the interpretation is that the effective number of functional groups (or species) are, respectively, 4, 3.60, and 3.33 with all pairwise distances at least 2 .

2) For a level of threshold distinctiveness $\tau=d_{\text {mean }}=$ 4.08 , which is the mean distance of any two randomly chosen individuals based on the original distance matrix, we have 


$$
\Delta(4.08)=\left[\begin{array}{cccc}
0 & 2 & 4.08 & 4.08 \\
2 & 0 & 4.08 & 4.08 \\
4.08 & 4.08 & 0 & 4.08 \\
4.08 & 4.08 & 4.08 & 0
\end{array}\right]
$$

In this case, because the distance between species 1 and 2 is less than the threshold level 4.08, some individuals of species 1 are functionally indistinct from species 2 , and vice versa; see the illustrative diagrams in Fig. 1. The abundance of the $f$-indistinct set of species 1 (Eq. 3c) is $a_{1}(\tau)=n_{1}+(1-2 / 4.08) n_{2}=27.65$ whereas the abundance of the corresponding set of species 2 is $a_{2}(\tau)=n_{2}+(1-2 / 4.08) n_{1}=25.20$. These two $f$-indistinct sets share some individuals. Species 3 is functionally equally distinct from the other species at level $\tau=4.08$, implying that the $f$-indistinct set of species 3 includes only its own individuals and its attribute contribution is still unity. A similar argument holds for species 4. That is, we have $a_{3}(\tau)=n_{3}=10$, and $a_{4}(\tau)=n_{4}=5$. The corresponding attribute contributions are $\left\{v_{i}(\tau)=\right.$ $\left.n_{i} / a_{i}(\tau), i=1,2,3,4\right\}=\{0.723,0.595,1,1\}$. Here, the attribute contributions for species 1 and species 2 are less than unity because neither is functionally distinct from all other species at the level 4.08. Dividing each $a_{i}(\tau)$ by the total abundance, we obtain the corresponding "relative" abundances $\left\{a_{i}(\tau) / n_{+} ; i=1,2,3,4\right\}=\{0.553$, $0.504,0.2,0.1\}$. The functional diversity of order $q$ given in Eq. $4 \mathrm{a}$ is simply the Hill number of the same order $q$ for an assemblage in which there are four sub-assemblages: the sub-assemblages consists of $0.723,0.595,1$, and 1 functional groups, and the corresponding relative abundances for each group are, respectively, 0.553, $0.504,0.2$, and 0.1 . The functional diversity (Eq. 4a) gives ${ }^{0} F D(\Delta(4.08))=3.32,{ }^{1} F D(\Delta(4.08))=2.70$, and ${ }^{2} F D(\Delta(4.08))=2.37$. Thus, for $q=0,1$, and 2, there are, respectively, 3.32, 2.70, and 2.37 functional groups with pairwise distances at least 4.08 .

3) The conventional species-equivalent formula corresponds to a level of threshold distinctiveness $\tau=d_{\max }=8$. At this level, no truncation is needed and all distances remain the same as the original ones

$$
\Delta(8)=\left[\begin{array}{llll}
0 & 2 & 8 & 8 \\
2 & 0 & 8 & 8 \\
8 & 8 & 0 & 6 \\
8 & 8 & 6 & 0
\end{array}\right]
$$

For $\tau=d_{\max }=8$, we have $d_{12}(\tau)=2<8$ and $d_{34}(\tau)=$ $6<8$. Thus, not only the $f$-indistinct sets for species 1 and species 2 share some individuals, but also the $f$-indistinct sets for species 3 and species 4 share some individuals; see the diagrams in Fig. 1. The attribute contributions for the four species are $\left\{v_{i}(\tau)\right.$; $i=1,2,3,4\}=\{0.640,0.500,0.889,0.667\}$ and the set of "relative" abundances becomes $\left\{a_{i}(\tau) / n_{+}\right.$; $i=1,2,3,4\}=\{0.625,0.600,0.225,0.150\}$. Here, all attribute contributions are less than unity because none of the four species are functionally equally distinct from all others at the level $\tau=10$. The attribute diversity gives ${ }^{0} F D(\Delta(8))=2.70, \quad{ }^{1} F D(\Delta(8))=2.29$, and ${ }^{2} F D(\Delta$ $(8))=2.04$. Thus, for $q=0,1$ and 2 , there are, respectively, 2.70, 2.29, and 2.04 functional groups with pairwise distances at least 8 .

4) Finally, if we consider a level of threshold distinctiveness $\tau$ greater than $d_{\max }$, say, $\tau=10$, then all distances remain the same as the original ones

$$
\Delta(10)=\left[\begin{array}{llll}
0 & 2 & 8 & 8 \\
2 & 0 & 8 & 8 \\
8 & 8 & 0 & 6 \\
8 & 8 & 6 & 0
\end{array}\right]
$$

In this case, all pairwise distances are less than the level $\tau=10$. Therefore, the $f$-indistinct set of a species includes some individuals of any other species; see the illustrative diagrams in Fig. 1. There are shared individuals among any two or more $f$-indistinct sets. The attribute contributions become $\left\{v_{i}(\tau) ; i=1,2,3,4\right\}=\{0.571,0.441$, $0.526,0.313\}$ with the "relative" abundances $\left\{a_{i}(\tau) /\right.$ $\left.n_{+} ; i=1,2,3,4\right\}=\{0.7,0.68,0.380,0.320\}$. Then, we have ${ }^{0} F D(\Delta(10))=1.85,{ }^{1} F D(\Delta(10))=1.76$, and ${ }^{2} F D(\Delta$ $(10))=1.69$. Thus, for $q=0,1$, and 2 , there are, respectively, $1.85,1.76$, and 1.69 functional groups with pairwise distances at least 10 .

5) When the level is increased, all the four $f$-indistinct sets become larger. As $\tau$ tends to infinity, nearly all individuals become functionally indistinct and thus all the four $f$-indistinct sets coincide, with each including all individuals of the assemblage. In this case, we have $\left\{a_{i}(\tau) / n_{+} ; i=1,2,3,4\right\}=\{1,1,1,1\} \quad$ and $\left\{v_{i}(\tau) ; i=1,2,3,4\right\}=\{0.4,0.3,0.2,0.1\}$, which gives ${ }^{q} F D(\Delta(\tau))=1$ for all $q \geq 0$, implying there is only one functional group.

\section{Functional Diversity Profiles}

Given a species-pairwise distance matrix and species abundances, our functional diversity (Eqs. 4a-4e) varies with diversity order $q \geq 0$ and the threshold distinctiveness level $\tau>0$. Thus, functional diversity is characterized by a three-dimensional surface that depicts the functional diversity as a function of $q$ and $\tau$. A plot of this three-dimensional surface for the above hypothetical four-species example is shown in Fig. 2a. However, when there are multiple assemblages, comparisons of several three-dimensional surfaces become complicated. We thus advocate the use of two profiles to characterize functional diversity:

1. The $q$ profile.-For a given threshold distinctiveness level $\tau$, the $q$ profile depicts the functional diversity ${ }^{q} F D$ $(\Delta(\tau))$ as a function of the diversity order $q \geq 0$. We suggest considering three levels separately: $\tau=d_{\text {min }}, d_{\text {mean }}$, and $d_{\text {max }}$; see Fig. 2 b. Each $q$ profile can be theoretically 
a) Three-dimensional functional diversity surface for the hypothetical four-species example

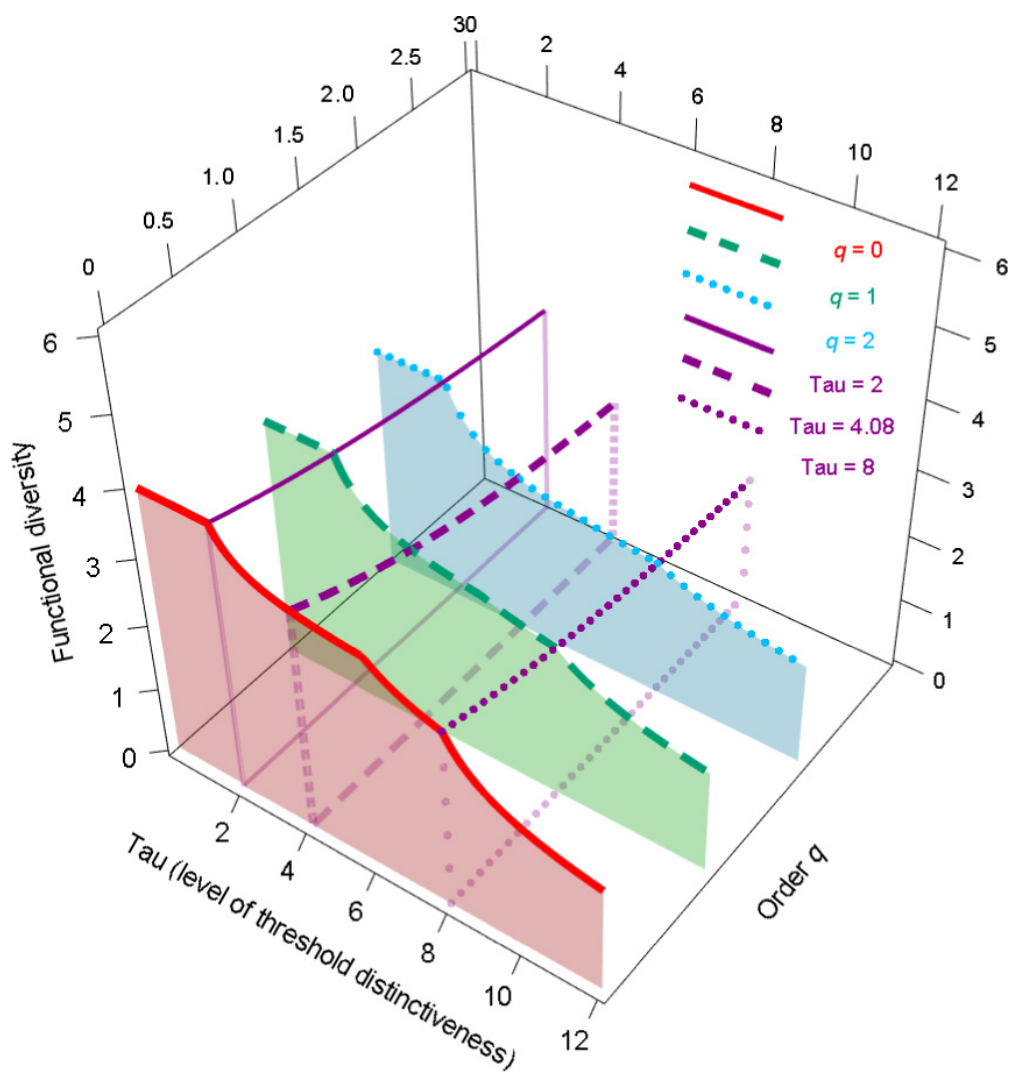

b) $q$-profile

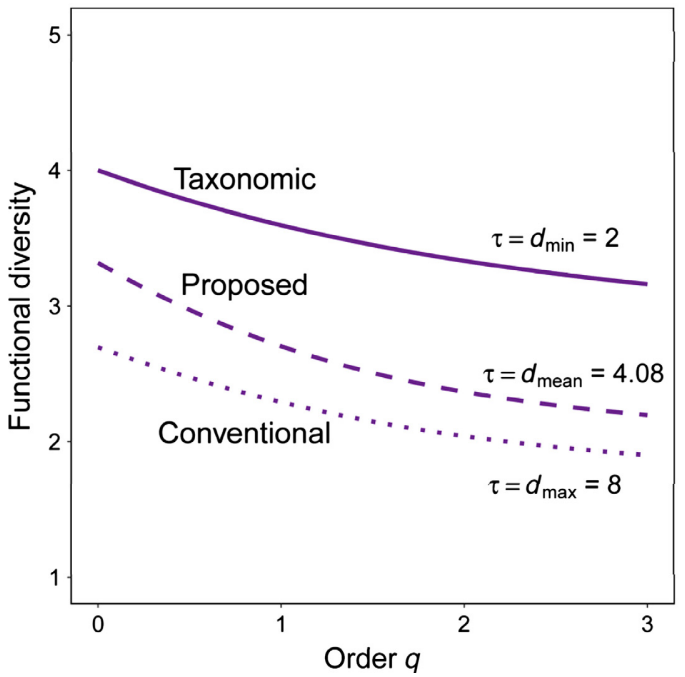

c) $\tau$-profile

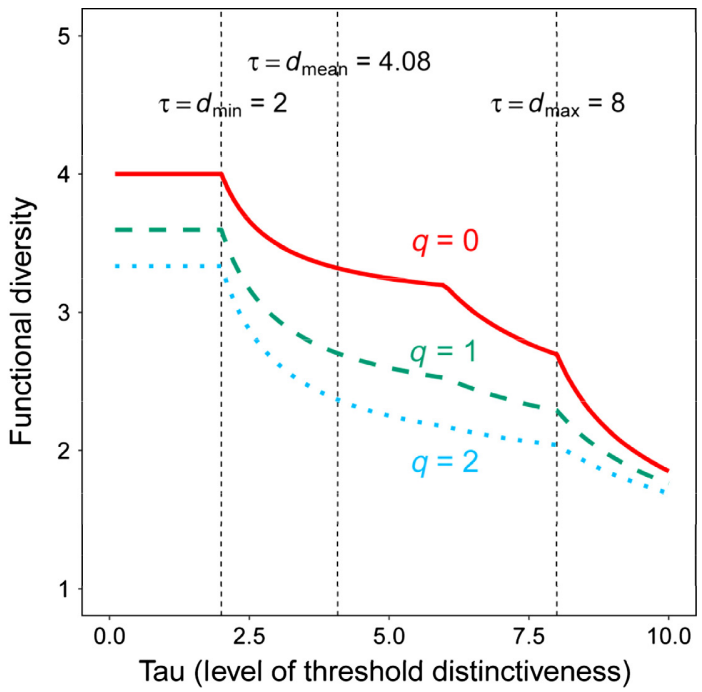

FIG. 2. (a) The three-dimensional functional-diversity surface, which plots ${ }^{q} F D(\Delta(\tau))$ as a function of diversity order $q \geq 0$ and level of threshold distinctiveness $\tau>0$. (b) The $q$ profile, which plots ${ }^{q} F D(\Delta(\tau))$ as a function of diversity order $q$ for three levels of threshold distinctiveness (minimum, mean, and maximum): $\tau=d_{\min }, d_{\text {mean }}$, and $d_{\max }$ for the four-species example described in the A simple hypothetical example section. The taxonomic diversity corresponds to the profile with $\tau=d_{\min }$ whereas the conventional functional diversity corresponds to the profile at $\tau=d_{\max }$. (c) The $\tau$ profile plots ${ }^{q} F D(\Delta(\tau))$ as a function of level of threshold distinctiveness $\tau$, for $q=0,1$, and 2. The three dotted vertical lines correspond to different levels of threshold distinctiveness. 
proved to be a non-increasing function of diversity order $q$. The $q$ profile for $\tau=d_{\min }$ represents the taxonomic diversity (Hill numbers) profile as a function of $q$; the steepness of the slope of this profile reflects the degree of unevenness among species relative abundances. Note that if two or more species have identical trait values, these species should be grouped into one functionally identical "species" in the assessment of taxonomic diversity and evenness. The $q$ profile for $\tau=d_{\max }$ represents the functional diversity profile based on the conventional species-equivalent formula. The $q$ profile for $\tau=d_{\text {mean }}$ represents our recommended functional diversity profile for comparing numerous assemblages, as discussed in later text. This profile is positioned between the two previously mentioned profiles, and the slope of this profile reflects functional unevenness or the degree of inequality among the abundances of functionally indistinct sets, weighted by the attribute contribution of each species.

2. The $\tau$ profile.-The $\tau$ profile depicts the functional diversity ${ }^{q} F D(\Delta(\tau))$ as a function of the threshold level $\tau$. Multiple assemblages can be compared with each other at the same level of $\tau$ for any fixed value of $q$. We suggest presenting the profiles for $q=0,1$, and 2 separately; see Fig. $2 \mathrm{c}$ for an example. Each $\tau$ profile can be theoretically proved to be a non-increasing function of $\tau$; it decreases from the species diversity (the leftmost level of the $\tau$ profile when $\tau \leq d_{\min }$ ) to unity when $\tau$ tends to infinity. In the infinity case, we hypothesize that the distance between two species could be very high so all species are actually far from being distinct at the specified high level. Thus, the assemblage is considered to host only one species-equivalent. The diversity value for any $\tau>0$ is always between 1 and the actual species richness. When $\tau=d_{\max }$, the profile value ${ }^{q} F D$ $\left(\Delta\left(d_{\max }\right)\right)$ reduces to the value based on the conventional formula in Eq. 5d, implying that the conventional functional diversity is incorporated into the proposed $\tau$ profile. As explained near Eq. 5d, unless there are several species pairs in the original distance matrix that have the value $d_{\max }$, the conventional formula often yields values close to unity especially when there are many species.

Other properties of the two types of profiles are listed in Appendix S2. To compare functional diversity across multiple assemblages, we compare their $\tau$ profiles over a proper range covering the interval $\left[0, d_{\max }\right]$ and their $q$ profiles over diversity order from $q=0$ to $q=2$ or 3; see Fig. 2b, c. In Appendix S2, we use examples to illustrate in more detail how our functional diversity measures are sensitive to the species abundances and species-pairwise distances.

From a practical point of view, examining profiles of dozens of assemblages to compare their functional diversity becomes challenging. Therefore, we suggest comparing the functional diversities for $q=0,1$, and 2 computed at $\tau=d_{\text {mean }}$ if there are many assemblages; this is similar to the reasoning behind phylogenetic diversity (Chao et al. 2010) based on a concept of "mean branch length." The proposed functional diversity of a given order $q$ with $\tau=d_{\text {mean }}$ could yield rankings among assemblages that are different to rankings from the conventional method with $\tau=d_{\max }$, because the conventional method is less sensitive to actual functional differences between assemblages than is our proposed measures. Hypothetical examples in Appendix S2 also reveal that our proposed functional diversity at the threshold distinctiveness $\tau=d_{\text {mean }}$ yields more reasonable results than the conventional formula at $\tau=d_{\max }$. An integrated measure that can simultaneously consider all plausible threshold levels is called AUC (area under the curve of a $\tau$ profile). Another justification for our choice of using $\tau=d_{\text {mean }}$ is that for this value of $\tau$, the proposed functional diversity generally yields rankings among assemblages that are highly consistent with rankings based on the AUC index. See Conclusion and discussion and Appendix S2 for other possible choices of $\tau$ and the comparison with the AUC index.

\section{Functional Diversity Decomposition}

Here, we show that the partitioning theory of attribute diversity (Chao et al. 2014a) can be applied to our functional diversity measure to obtain alpha, beta, and gamma diversity measures for a set of $N$ assemblages. Assume that there are $S$ species in the pooled assemblage. Denote the pairwise distance matrix of these $S$ species by $\left[d_{i j}\right]$. For any given level of threshold distinctiveness $\tau>0$, define the truncated distance matrix $\Delta$ $(\tau) \equiv\left[d_{i j}(\tau)\right]=\left[\min \left(d_{i j}, \tau\right)\right]$ as in Eq. $3 \mathrm{~b}$.

To present functional diversity decomposition, we need to generalize the one-assemblage notation to multiple assemblages. Define $n_{i k}$ as the number of individuals (or biomass, percent cover, or any other measure of species abundance) of the $i$ th species in the $k$ th assemblage, $i=1,2, \ldots, S, k=1,2, \ldots, N$. Some of the $n_{i k}$ may be zero. Let $n_{+k}=\sum_{i=1}^{S} n_{i k}$ be the size of the $k$ th assemblage, $n_{i+}=\sum_{k=1}^{N} n_{i k}$ be the total abundances of the $i$ th species in the pooled assemblage, and $n_{++}=$ $\sum_{k=1}^{N} n_{+k}=\sum_{i=1}^{S} n_{i+}$ be the total abundance in the pooled assemblage. Denote $a_{i k}(\tau)=\sum_{j=1}^{S}\left[1-f\left(d_{i j}(\tau)\right)\right]$ $n_{j k}$ as the abundance of the (level- $\tau$ ) functionally indistinct set of species $i$ in the $k$ th assemblage. The total number of individuals in the pooled assemblage that are functionally indistinct from species $i$ is $a_{i+}(\tau)=$ $\sum_{k=1}^{N} a_{i k}(\tau)=\sum_{j=1}^{S}\left[1-f\left(d_{i j}(\tau)\right)\right] n_{j+}$.

The attribute contribution (the proportional number of functional groups contributed by species $i$ in the pooled assemblage) is $v_{i+}(\tau)=n_{i+} / a_{i+}(\tau)$, as we explained in the case of an individual assemblage. The attribute-contribution-weighted sum of these abundances is expressed as $\bar{V}(\tau)=\sum_{i=1}^{S} v_{i+}(\tau) a_{i+}(\tau)=\sum_{i=1}^{S} n_{i+}=n_{++} . \quad$ Applying the functional-diversity formula (Eq. 4a) to the pooled assemblage, we obtain the functional gamma diversity 
${ }^{q} F D_{\gamma}(\Delta(\tau))=\left(\sum_{i=1}^{S} v_{i+}(\tau)\left(\frac{a_{i+}(\tau)}{n_{++}}\right)^{q}\right)^{1 /(1-q)}, q \geq 0, q \neq 1$.

When $q$ tends to $1,{ }^{q} F D_{\gamma}(\Delta(\tau))$ has the limit

$$
\begin{aligned}
{ }^{1} F D_{\gamma}(\Delta(\tau)) & =\lim _{q \rightarrow 1}^{q} F D_{\gamma}(\Delta(\tau)) \\
& =\exp \left(-\sum_{i=1}^{S} v_{i+}(\tau) \frac{a_{i+}(\tau)}{n_{++}} \log \frac{a_{i+}(\tau)}{n_{++}}\right) .
\end{aligned}
$$

The functional gamma diversity is interpreted as the effective number of functionally equally distinct species (or virtual functional groups) in the pooled assemblage with the distance between any two species-equivalents at least $\tau$.

Note that the species in each individual assemblage are a subset of those in the pooled assemblage, and thus, the distance matrix of each individual assemblage is a subset of the matrix $\left[d_{i j}\right]$ for the pooled assemblage. That is, all computations could be done only once for the global functional distance matrix; the functional diversity of each individual assemblage can be simply computed from the matrix of the pooled assemblage. Based on the decomposition theory of Chao et al. (2014a), the alpha attribute diversity (functional alpha diversity) is a function of the abundances $a_{i k}(\tau)$ and the attribute contributions determined in the pooled assemblage

$$
\begin{aligned}
{ }^{q} F D_{\alpha}(\Delta(\tau))= & \frac{1}{N}\left(\sum_{k=1}^{N} \sum_{i=1}^{S} v_{i+}(\tau)\left(\frac{a_{i k}(\tau)}{n_{++}}\right)^{q}\right)^{1 /(1-q)} \\
& \geq 0, q \neq 1
\end{aligned}
$$

Here, if $a_{i k}(\tau)=0$, then we define $0^{0} \equiv 0$. When $q$ tends to $1,{ }^{q} F D_{\alpha}(\Delta(\tau))$ has the limit

$$
\begin{aligned}
{ }^{1} F D_{\alpha}(\Delta(\tau)) & =\lim _{q \rightarrow 1}^{q} F D_{\alpha}(\Delta(\tau)) \\
& =\frac{1}{N} \exp \left(-\sum_{i=1}^{S} \sum_{k=1}^{N} v_{i+}(\tau) \frac{a_{i k}(\tau)}{n_{++}} \log \frac{a_{i k}(\tau)}{n_{++}}\right) .
\end{aligned}
$$

The functional alpha diversity is interpreted as the effective number of functionally equally distinct species (or virtual functional groups) per assemblage with the distance between any two species-equivalents at least $\tau$.

Using a multiplicative partitioning, we obtain the functional beta diversity at the threshold distinctiveness level of $\tau$ as the ratio of gamma diversity to alpha diversity

$$
{ }^{q} F D_{\beta}(\Delta(\tau))=\frac{{ }^{q} F D_{\gamma}(\Delta(\tau))}{{ }^{q} F D_{\alpha}(\Delta(\tau))} .
$$

To describe the properties of the functional beta diversity, we first define $N$ assemblages to be functionally identical for any given level $\tau>0$, if the abundances of the $S$ functionally indistinct sets are identical across the $N$ assemblages, i.e., $a_{i 1}(\tau)=a_{i 2}(\tau)=\ldots=a_{i N}(\tau)$ for any species $i=1,2, \ldots, S$. Intuitively, this definition means that the abundance distributions over all possible trait profiles are identical across the $N$ assemblages, regardless of whether species are shared or not among assemblages. To illustrate this, consider an extreme example. Suppose species are described using two traits: Trait 1 can take three values $(t 1, t 2, t 3)$ and Trait 2 can take two values $(b 1, b 2)$. Then there are six possible trait combinations, i.e., $\{(t 1, b 1),(t 1, b 2),(t 2, b 1),(t 2, b 2),(t 3, b 1)$, $(t 3, b 2)\}$. In this case, two assemblages are functionally identical if the numbers of individuals in the six combinations are the same across both assemblages; here, the species identities in the two assemblages can be identical, partially shared or even completely non-shared. Moreover, since different species may have the same trait values, functionally identical assemblages may even have different numbers of species. In the special case that all species are equally distinct from each other, we have $a_{i k}(\tau)=n_{i k}$, for all $i=1,2, \ldots, S$ and $k=1,2, \ldots, N$. Then, our definition reduces to that for taxonomic diversity, i.e., $N$ assemblages are taxonomically identical if the raw abundance distributions are identical across all assemblages.

In Appendix S3, we proved that the proposed functional gamma diversity is never less than the alpha diversity. The multiplicative beta diversity is always between the minimum value of unity (when all assemblages are functionally identical) and the maximum value of $N$ (when there are no shared species among the $N$ assemblage and any two species from two different assemblages have a distance at least $\tau$ ) regardless of the values of alpha and gamma. Thus, alpha and beta are independent (Chao and Chiu 2016). The beta diversity can be interpreted as the effective number of equally large assemblages with no shared species, where all species are equally distinct from each other and species-pairwise distances are at least $\tau$. The two types of diversity profiles can be similarly obtained for functional beta diversity. When all species are functionally equally distinct at a given level of threshold distinctiveness, our functional beta diversity thus reduces to the abundance-based beta diversity derived in Chiu et al. (2014).

The decomposition of quadratic entropy has been discussed in the literature (e.g., Ricotta and Szeidl 2006). The conventional species-equivalent formula of the gamma quadratic entropy based on Eq. $5 \mathrm{c}$ is identical to our gamma diversity of $q=2$ and $\tau=$ global maximum distance (the maximum distance in the pooled assemblage). However, our diversity decomposition is different from the conventional partitioning. Consequently, our alpha and beta diversity measures are different from those based on the conventional approach. This can be understood by noting that the conventional gamma may 
be less than alpha if the square-root-of-distance matrix is not Euclidean, whereas our gamma is never less than alpha for any type of distance matrix.

\section{Functional Dissimilarity Profiles}

Because the range of the multiplicative beta components depends only on $N$, following Chiu et al. (2014) and Chao et al. (2014a), we can transform the beta diversity to obtain four classes of (dis)similarity measures in the range $[0,1]$, as described below. Chiu et al. (2014) proposed four classes of taxonomic/phylogenetic overlap/similarity measures $\left(C_{q N}, U_{q N}, S_{q N}\right.$, and $\left.V_{q N}\right)$ and their corresponding complementary dissimilarity measures. Below we present the functional versions. Note that in the following interpretation of each functional dissimilarity measure, all speciesequivalents are assumed to be functionally equally distinct with the distance between any two speciesequivalents at least $\tau$.

Sørensen-type functional non-overlap measure in the range $[0,1]$. -

$$
1-C_{q N}(\Delta(\tau))=\frac{1-\left[{ }^{q} F D_{\beta}(\Delta(\tau))\right]^{1-q}}{1-N^{1-q}}, q \geq 0, q \neq 1 .
$$

This class of measures quantifies a property from the perspective of a single assemblage, i.e., it quantifies the effective average proportion of non-shared species in an assemblage. The "effective" average proportion is understood in the sense of being the "true non-overlap" fraction in a single assemblage. That is, this class of measures satisfies the following property: if each of the $N$ assemblages has $S$ equally common and equally distinct species with the distance between any two speciesequivalents at least $\tau$, and there are $A$ species shared by all of the assemblages and the remaining species in each assemblage are not shared with any other assemblages, then for all $q \geq 0$ the Sørensen-type non-overlap measure always gives the non-shared fraction, $1-A / S$, of each assemblage.

Jaccard-type functional non-overlap measure in the range $[0,1]$ - -

$$
1-U_{q N}(\Delta(\tau))=\frac{1-\left[{ }^{q} F D_{\beta}(\Delta(\tau))\right]^{q-1}}{1-N^{q-1}}, q \geq 0, q \neq 1 .
$$

This class of measures quantifies a property from the perspective of the pooled assemblage, i.e., it quantifies the proportion of non-shared species in the pooled assemblage. The "effective" proportion is understood in the sense of being "true non-overlap" fraction in the pooled assemblage. That is, this class of measures satisfies the following property: if there are $S$ equally common and equally distinct species with the distance between any two species-equivalents at least $\tau$ in the pooled assemblage, and there are exactly $R$ species shared by all $N$ assemblages and the remaining $S-R$ unshared species are evenly distributed across the $N$ assemblages, then for all $q \geq 0$, the Jaccard-type nonoverlap measure always gives the non-shared fraction, 1 $-R / S$, of the pooled assemblage. For $q=1$, the limits of the above two measures are identical

$$
1-C_{1 N}(\Delta(\tau))=1-U_{1 N}(\Delta(\tau))=\frac{\log \left[{ }^{1} F D_{\beta}(\Delta(\tau))\right]}{\log N} .
$$

Sørensen-type functional turnover measure in the range $[0,1]$. -

$$
1-V_{q N}(\Delta(\tau))=\frac{{ }^{q} F D_{\beta}(\Delta(\tau))-1}{N-1} .
$$

This measure quantifies the normalized species turnover rate in an assemblage (i.e., relative to alpha) because it can be expressed as $(N-1)^{-1}(\gamma-\alpha) / \alpha$; the constant $(N-1)^{-1}$ is used to normalize this measure to the range $[0,1]$. When $q=0,1-V_{0 N}(\Delta(\tau))=$ $1-C_{0 N}(\Delta(\tau))$; for $q=2$, we have $1-V_{2 N}(\Delta(\tau))=$ $1-U_{2 N}(\Delta(\tau))$. However, for $q=1$, this measure does not relate to any of our other functional dissimilarity measures.

Jaccard-type functional turnover measure in the range $[0,1]$. -

$$
1-S_{q N}(\Delta(\tau))=\frac{1-1 /\left[{ }^{q} F D_{\beta}(\Delta(\tau))\right]}{1-1 / N}
$$

This measure quantifies the normalized species turnover rate with respect to the pooled assemblage (i.e., relative to gamma) because it can be expressed as $(1-1 /$ $N)^{-1}(\gamma-\alpha) / \gamma ;$ the constant $(1-1 / N)^{-1}$ is used to normalize this measure to the range $[0,1]$. For $q=0$, $1-S_{0 N}(\Delta(\tau))=1-U_{0 N}(\Delta(\tau))$; for $q=2,1-S_{2 N}$ $(\Delta(\tau))=1-C_{2 N}(\Delta(\tau))$. However, for $q=1$, this measure does not relate to any of the above measures.

Each of the four functional dissimilarity measures above ranges from 0 (when all assemblages are functionally identical) to unity (when there are no shared species among the $N$ assemblages and any two species from two different assemblages have a distance at least $\tau$ ). Therefore, each measure can be compared across multiple sets of assemblages. When the actual trait-based distances of all different-species pairs are at least $\tau$ in an assemblage, each of the functional dissimilarity measures above reduces to its corresponding taxonomic version (Chiu et al. 2014). Because the two classes of functional 
turnover measures for $q=0$ and 2 are special cases of the functional non-overlap measures, we will mainly focus on the Sørensen- and Jaccard-type non-overlap measures $1-U_{q N}(\Delta(\tau))$ and $1-C_{q N}(\Delta(\tau))$ in our analyses of real data.

As in the one assemblage case, we also propose two types of (dis)similarity profiles ( $\tau$ profile and $q$ profile) for each of the two dissimilarity measures: $1-U_{q N}(\Delta(\tau))$ and $1-C_{q N}(\Delta(\tau))$. When $\tau=d_{\max }$, the dissimilarity measures often yield low values, implying that assemblages are highly functionally similar with only one or a small number of functional groups. See the real data analysis for examples. We emphasize that the low dissimilarity may be genuinely true for some special data sets, but in other data sets these conventional measures necessarily yield low values as a consequence of setting a maximum level of threshold distinctiveness.

\section{Real Examples}

\section{Beetle data}

The abundance data presented here focus on saproxylic beetles collected over two years on trees in a mountain forest ecosystem (analysed and discussed in Thorn et al. 2016). The design consists of 12 plots each composed of three experimentally felled trees, resulting in a total of 36 experimental felled trees. In each plot, the bark of one tree was completely removed, the bark of a second tree was only partially removed (i.e., barkscratched), and the third tree served as a control. The design is thus composed of 12 replications of three different treatments (i.e., control, bark-scratched, and debarked). A total of 120 species of saproxylic beetles were trapped with emergence traps on felled trees (Thorn et al. 2016). There were 84 species (11,346 individuals) trapped in the control trees, 86 species $(10,716$ individuals) in the bark-scratched trees, 61 species (3,201 individuals) in the debarked trees, with 43 species shared by the three treatments. Our analysis was based on the species relative abundance data; see Conclusion and discussion for some remarks regarding the use of raw abundance data vs. relative abundance data.

All species were characterized for a set of 10 functional traits (Seibold et al. 2015), five of them continuous: body size, mean elevation above sea level, mean wood diameter, decay stage of dead wood, and canopy cover of forests in which a species occurs. Categorical traits included three binary traits (flower visiting, coniferous host trees, and broad-leaved host trees), and two traits with three categories each: microhabitat guild (wood-bark, cavities, fungi) of larvae, and feeding type (mycetophagous, xylophagous, zoophagous). Speciesby-species distances were computed by means of a Gower-distance with equal weighting on each trait except for the two binary host tree traits, which were each weighted 0.5 each (Gower 1971). Since the Gower distance takes values between 0 and 1 , the level of threshold distinctiveness $\tau$ can be any positive value between 0 and 1 .

We assessed functional diversity within each of the three treatments based on two types of two-dimensional profiles. For each of the three treatments, Fig. 3 shows the $q$ profile for $0 \leq q \leq 2$ separately for $\tau=d_{\text {min }}$ (0.0008), $d_{\text {mean }}(0.1019)$, and $d_{\max }(0.7105)$ in the upper panels (Fig. 3a-c), and the $\tau$ profile for $0<\tau<0.4$ (Fig. 3d-f, all curves become flat after $\tau=0.4$ ) separately for $q=0,1$, and 2 in the lower panels. Here, $d_{\min }$, $d_{\text {mean }}$, and $d_{\max }$ refer to the minimum, mean, and maximum of the species pairwise distances in the set of 120 species in the pooled assemblage. Details of diversity values for the combinations of three diversity orders of $q$ $(0,1$, and 2$)$ and three threshold distinctiveness levels of $\tau\left(d_{\text {min }}, d_{\text {mean }}\right.$, and $\left.d_{\text {max }}\right)$ are provided in Appendix S4: Table S1.

The $q$ profile for $\tau=d_{\min }$ (Fig. 3a) reveals that taxonomic diversities in the control trees and bark-scratched trees are very close to each other; both diversities are higher than the diversity of debarked trees, especially for rare species (for $q=0$, there were, respectively, 84, 86, and 61 species for control, scratched, and debarked trees), though the difference diminishes when our focus is shifted to common species or dominant species (the corresponding diversity values for the three treatments are $9.81,10.20$, and 8.32 for $q=1$ ). This was probably due to the loss of many phloem-feeding and other mostly rare species associated with bark. The steep slope in each of the $q$ profiles in Fig. 3a reflects substantial unevenness of species abundances.

For the conventional approach $\left(\tau=d_{\max }=0.7105\right.$, Fig. 3c), the $q$ profile shows that all functional diversities are close to unity. Setting a threshold distinctiveness level of unity leads to functional diversity values even closer to unity, implying only one species-equivalent in each treatment and yielding almost identical functional diversities among the three treatments. Our proposed approach (Fig. $3 b, \tau=d_{\text {mean }}$ ) reveals that the pattern for functional diversity is similar to that of taxonomic diversity: the two curves representing bark-scratched and control trees closely match each other, with both being higher than the curve representing debarked trees. As with taxonomic diversity, the magnitude of the difference decreases with diversity order $q$, i.e., 27.24 for control trees, 29.42 for scratched trees, 19.74 for debarked trees for $q=0$, whereas the corresponding values are $4.30,4.69$, and 3.75 for $q=1$. A consistent pattern holds for any other threshold level as shown in the three $\tau$ profiles (Fig. $3 d-$ f). Note that the sharp decrease in each of the three $q$ profiles in Fig. 3b implies that the abundances of functionally indistinct sets are highly uneven, regardless of treatment.

All of these findings signify that functional diversity within the control and bark-scratched trees is lost with debarking. For rare species ( $q$ is small), the loss is pronounced, whereas for common species $(q=1)$ or dominant species $(q=2)$, the loss is limited. Consequently, 


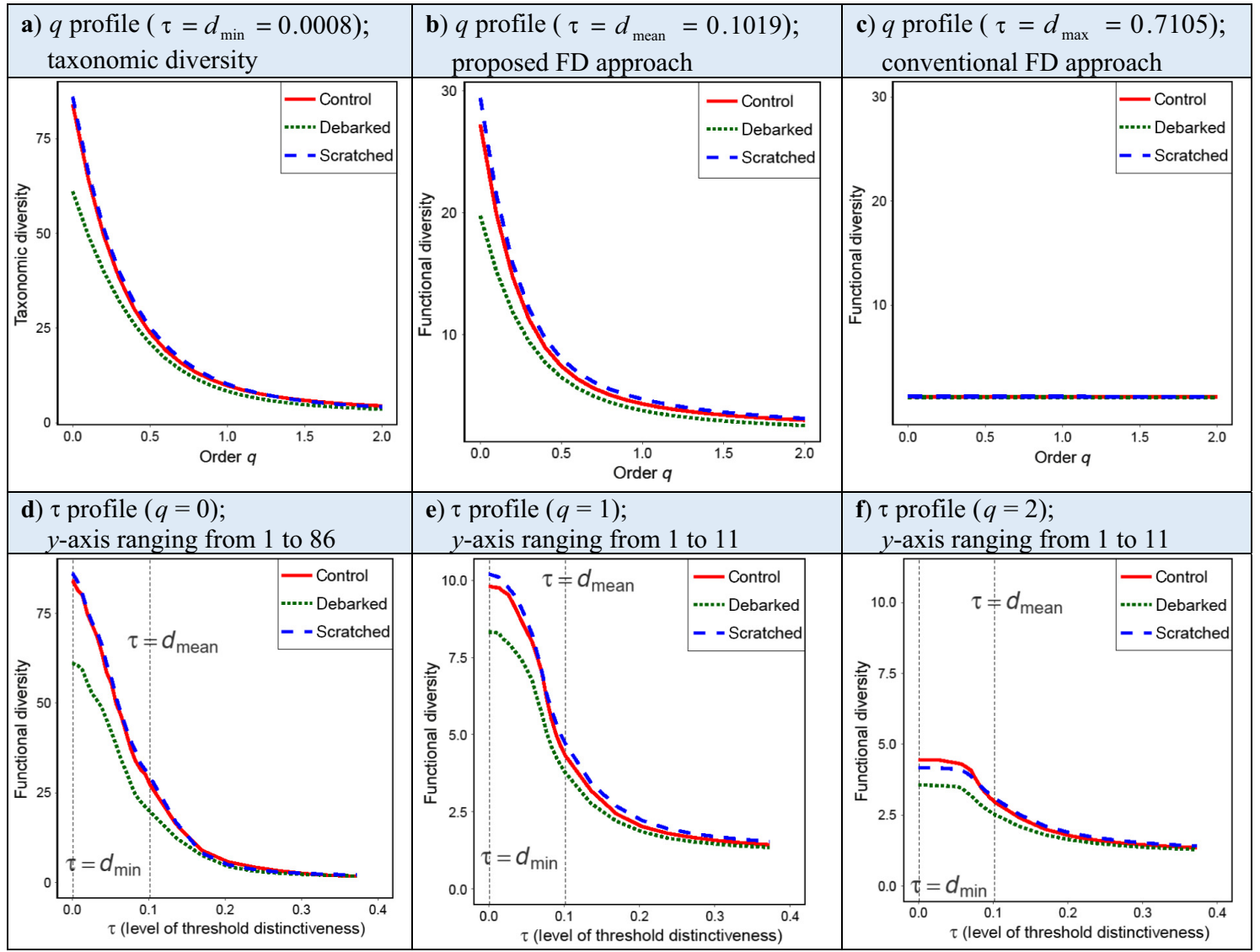

FIG. 3. The within-treatment functional diversity profile for three bark treatments (control, debarked, bark scratched) as a function of diversity order $q, 0 \leq q \leq 2$ (panels a, b, c), and as a function of threshold distinctiveness level $\tau, 0<\tau<0.4$ (panels $\mathrm{d}$, e, f), based on beetle species relative abundance data (Thorn et al. 2016). The two dotted vertical lines in the lower panels refer to two different levels of threshold distinctiveness: $d_{\min }(0.0008)$ and $d_{\text {mean }}(0.1019)$, the minimum and mean species-pairwise distances in the pooled assemblage. The curves are truncated at 0.4 as the diversity values remain at a constant when $\tau>0.4$, and thus, the diversity value for $d_{\max }(0.7105)$ is not shown.

rare species contribute disproportionally to high functional diversity in the control and bark-scratched trees. This pattern arises mainly due to the fact that barkscratched and control trees host species with a wide range of different functions, whereas debarked trees host only a subset, such as saproxylic species that bore into heartwood (Thorn et al. 2016). Thus, our measures provide new insights that the conventional approach does not.

To assess the among-treatment functional differentiation, we recommend two types of functional dissimilarity profiles based on the two measures: the Sørensentype functional dissimilarity measure $1-C_{q N}(\Delta(\tau))$ (Eq. 9a), and the Jaccard-type functional dissimilarity measure $1-U_{q N}(\Delta(\tau))$ (Eq. 9b). Fig. 4 provides such profiles for the trio of treatments. Some specific dissimilarity values for the combinations of three diversity orders $(q=0,1$, and 2$)$ and three threshold distinctiveness levels $\left(\tau=d_{\text {min }}, d_{\text {mean }}\right.$, and $\left.d_{\max }\right)$ are provided in Appendix S4: Table S1.
For species compositional differentiation (the $q$ profiles for $\tau=d_{\min }$ in Fig. 4a, b), the three bark treatments yield high taxonomic dissimilarity values. In contrast, the conventional functional dissimilarity based on the value $\tau=d_{\max }$ (the $q$ profiles for $\tau=d_{\max }$ in Fig. 4a, b) show very low (near 0 ) functional dissimilarity measures among the three treatments. Such low differentiation highlights the consequence of setting a very high level of threshold distinctiveness when looking to detect differentiation among the three treatments. The low differentiation reflects this extreme level of threshold distinctiveness.

The proposed $q$ profile with $\tau=d_{\text {mean }}=0.1019$ (Fig. 4a, b) lies between the two profiles with $\tau=d_{\min }$ and $\tau=d_{\max }$ respectively. For $q=0$ (functional richness), we have the Sørensen-type dissimilarity value of $1-C_{03}(\Delta(\tau))=0.06$, and the Jaccard-type dissimilarity value of $1-U_{03}(\Delta(\tau))=0.160$ among the three treatments (Appendix S4: Table S1). This can be interpreted in an effective sense to mean that about $6 \%$ of each 


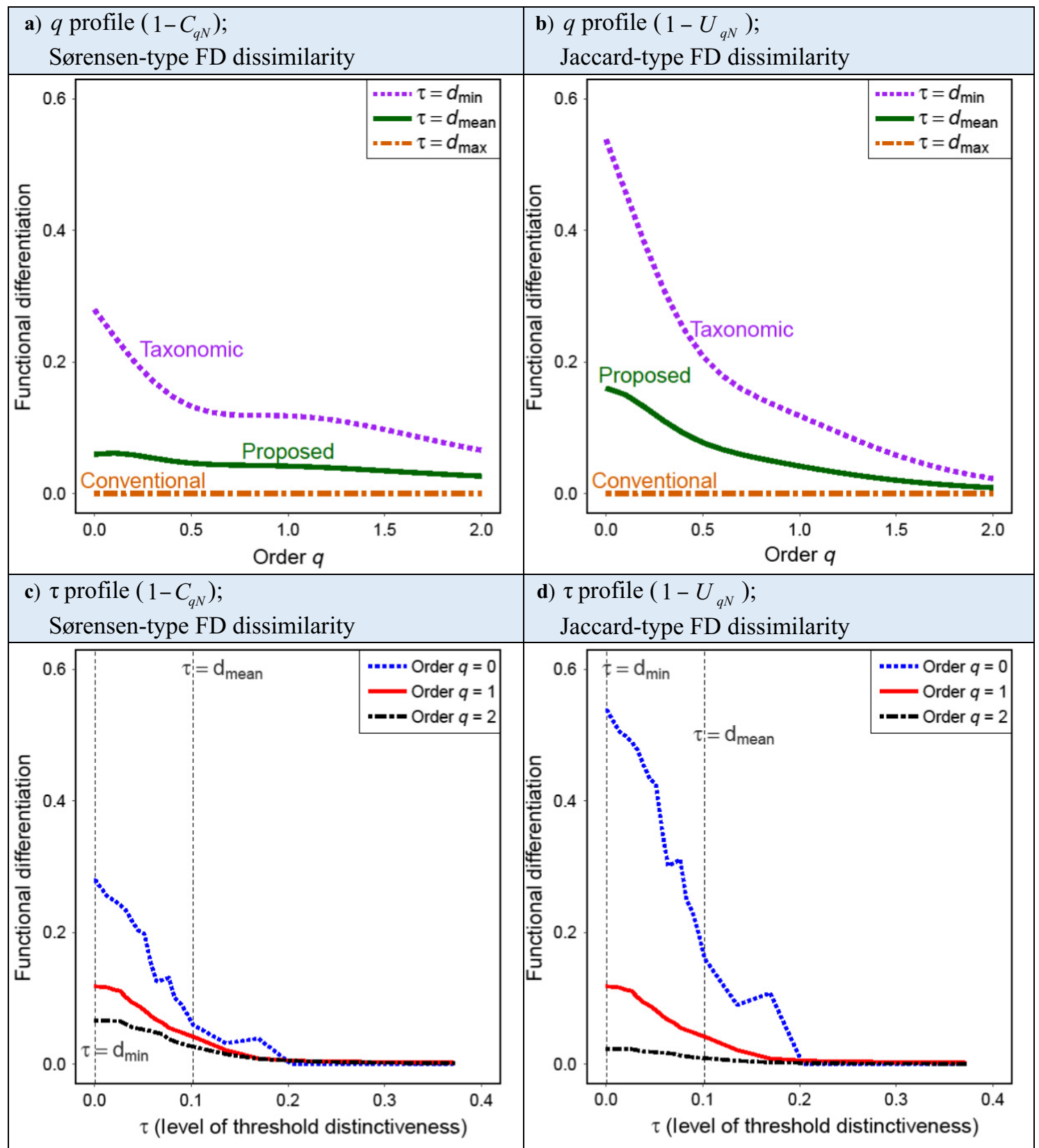

FIG. 4. The among-treatment (a, c) Sørensen-type (Eq. 9a) and (b, d) Jaccard-type (Eq. 9b) functional differentiation/dissimilarity profiles among the three bark treatments (control, debarked, bark-scratched) as a function of diversity order $q, 0 \leq q \leq 2$ (panels a, b), or as a function of threshold distinctiveness level $\tau, 0<\tau<1$ (panels $\mathrm{c}, \mathrm{d}$ ), based on beetle species relative abundance data (Thorn et al. 2016). See Fig. 3 for the meaning of the vertical dotted lines in the lower panels and the values of $d_{\text {min }}$ and $d_{\text {mean }}$. The curves are truncated at 0.4 as the diversity values remain at a constant when $\tau>0.4$, and thus, the dissimilarity value for $\tau=d_{\max }(0.7105)$ is not shown.

treatment's functional groups are not shared by the other two treatments, and about $16 \%$ of the pooled assemblage's functional groups are not shared by all treatments. These two dissimilarity values decline with $q$, implying that the dissimilarity among the three treatments is mainly attributed to rare species, a finding that is also revealed by the two $\tau$ profiles in Fig. $4 c$, d, where the curve for functional dissimilarity of $q=0$ lies above the other two curves for common species $(q=1)$ and dominant species $(q=2)$. 


\section{Woody plant data}

Our second example data set is based on woody plant censuses collected from two distinct forest ecosystems in Taiwan, the Fushan Forest Dynamics Plot $(500 \times 500 \mathrm{~m}, 25 \mathrm{ha})$ and the Kenting Forest Dynamics Plot $(400 \times 250 \mathrm{~m}, 10 \mathrm{ha})$. The two censuses were conducted in 2013. All woody stems greater than or equal to $1 \mathrm{~cm}$ diameter at breast height (DBH) in each plot were mapped, tagged, measured, and identified to species. For simplicity, these two plots are referred to as Fushan (FS) and Kenting (KT), respectively.

FS is located in the subtropical evergreen broadleaf forest of northern Taiwan $\left(24^{\circ} 45^{\prime} 40^{\prime \prime} \mathrm{N}, 121^{\circ} 33^{\prime} 28^{\prime \prime} \mathrm{E}\right)$, with an elevation ranging from 600 to $733 \mathrm{~m}$ above sea level. Annual rainfall is $4,271 \mathrm{~mm}$, with an average temperature of $18.2^{\circ} \mathrm{C}$ and a high mean relative humidity of $95.1 \%$. This forest experiences the Northeast monsoon in the winter and frequent typhoons during summer and autumn. KT is located in a karst forest in southern Taiwan $\left(21^{\circ} 57^{\prime} 58^{\prime \prime} \mathrm{N}, 120^{\circ} 49^{\prime} 7^{\prime \prime} \mathrm{E}\right)$, with an elevation ranging from 250 to $300 \mathrm{~m}$ above sea level. This forest is characterized by karst topography underlain by coral reef limestone. Annual rainfall is approximately $2,000 \mathrm{~mm}$, with an average temperature of $25.4^{\circ} \mathrm{C}$ and a mean relative humidity of $74.4 \%$. There is a dry season of 6 months (October-April), and soils are mainly composed of calcareous, neutral loam or light clay. See Su et al. (2007) and Wu et al. (2011) for detailed descriptions of the two plots.

We divided each plot into $50 \times 50 \mathrm{~m}$ quadrats for a total of 100 quadrats (FS) and 40 quadrats (KT). We randomly chose 40 quadrats from FS as representative data to compare with KT. Here, we only report the results based on the data of one random trial because the data for other trials were generally consistent.

A total of 94 and 86 species were recorded in the 10-ha data from FS and KT, respectively. Collectively, there were a total of 168 species in the pooled assemblage, of which $12(7.1 \%)$ species were shared between the two plots. FS is dominated by two species: Blastus cochinchinensis $(17.6 \%$ of the total individuals) and Helicia formosana (16.1\%). KT has a super-dominant species Diospyros maritima $(55.9 \%)$, and a second dominant species Drypetes littoralis (9.6\%); the most dominant five species accounted for approximately $74.8 \%$ of the total individuals.

Our comparison used three leaf traits from each species, specific leaf area (SLA), leaf dry-matter content (LDMC), and leaf thickness. These traits relate to niche variation, resource acquisition, and life history tradeoffs between photosynthetic rate and the cost of leaf growth. The functional distance between any two species was calculated based on the Gower-distance with equal weights for each trait.

As with the beetle abundance data, we compared within-plot diversities of the woody plants by constructing Fig. 5, which shows the corresponding $q$ profile (in the upper panels) separately for $\tau=d_{\min }(0.0035), d_{\text {mean }}$
(0.1556) and $d_{\max }(0.6077)$, and also the $\tau$ profile (in the lower panels) separately for $q=0,1$, and 2 . Some specific diversity values are provided in Appendix S4: Table S2. Congruent with the findings associated with the beetle data, the $q$ profile for $\tau=d_{\max }$ (Fig. 5c) shows that the conventional functional diversity values are all close to unity, and the two curves are almost indistinguishable. The $q$ profile for $\tau=d_{\min }$ (Fig. 5a) demonstrates that FS has a higher taxonomic diversity for all values of $q$ due to its higher species richness and higher degree of evenness among species abundances. Note that the sharper decline in KT's $q$ profile (Fig. 5a) implies that species abundances are more uneven in $\mathrm{KT}$, mainly due to the presence of a super-dominant species.

Fig. $5 \mathrm{~b}$ (for $\tau=d_{\text {mean }}$ ) shows that the proposed $q$ profiles of the two plots intersect at $q=0.7$. That is, when our focus is on common and dominant species $(q>0.7)$, FS has a higher functional diversity, in addition to a higher taxonomic diversity for all values of $q$. However, for rare species $(q<0.7)$, the ordering is reversed in that KT has a higher functional diversity. Our data provide an example showing that two functional $q$ profiles may intersect and that the functional diversity may exhibit opposite ordering with taxonomic diversity (in our case, when $q<0.7$ ). This intersection can be explained by the fact that, compared to FS, KT has greater functional richness but a lower degree of functional evenness. Note that the $q$ profile for FS declines very slowly (Fig. 5b) and is generally flat when $q>0.3$, whereas the slope of KT's $q$ profile is steep, i.e., the abundances of functionally indistinct sets are highly uneven. In other words, the presence of a super-dominant species in KT causes not only taxonomic unevenness but also functional unevenness in the data. As shown by the three $\tau$ profiles in the lower panels, KT possesses higher functional richness $\left(q=0\right.$, Fig. 5d) for nearly all threshold levels $>d_{\min }$. However, when abundances are considered $(q=1$ and $q=2$, Fig. 5e, f), the effect of the super-dominant species leads to lower functional diversity in KT.

The fact that FS and KT exhibit different ordering patterns in taxonomic and functional diversities may reflect the nature of their respective forest types. Higher functional richness $(q=0)$ in KT (Fig. 5b, d) may arise from its contrasting microhabitats. KT is characterized by a karst terrain, which includes alterations of limestone outcrops and depressions. Limestone outcrops are characterized by thin soils and low levels of soil moisture, while soils in the depression areas can be thick, fertile and poorly drained. Such sharp contrasts in microhabitats may select species with contrasting leaf traits, and many rare species are habitat specialists that only occurred on these specific microhabitats. On the other hand, the fact that one super-dominant species (D. maritima) plus the five most dominant species made up $75 \%$ of total individuals in KT may largely explain why lower abundance-based functional diversity was found in common and dominant species in this habitat (Fig. 5b, e, f). 


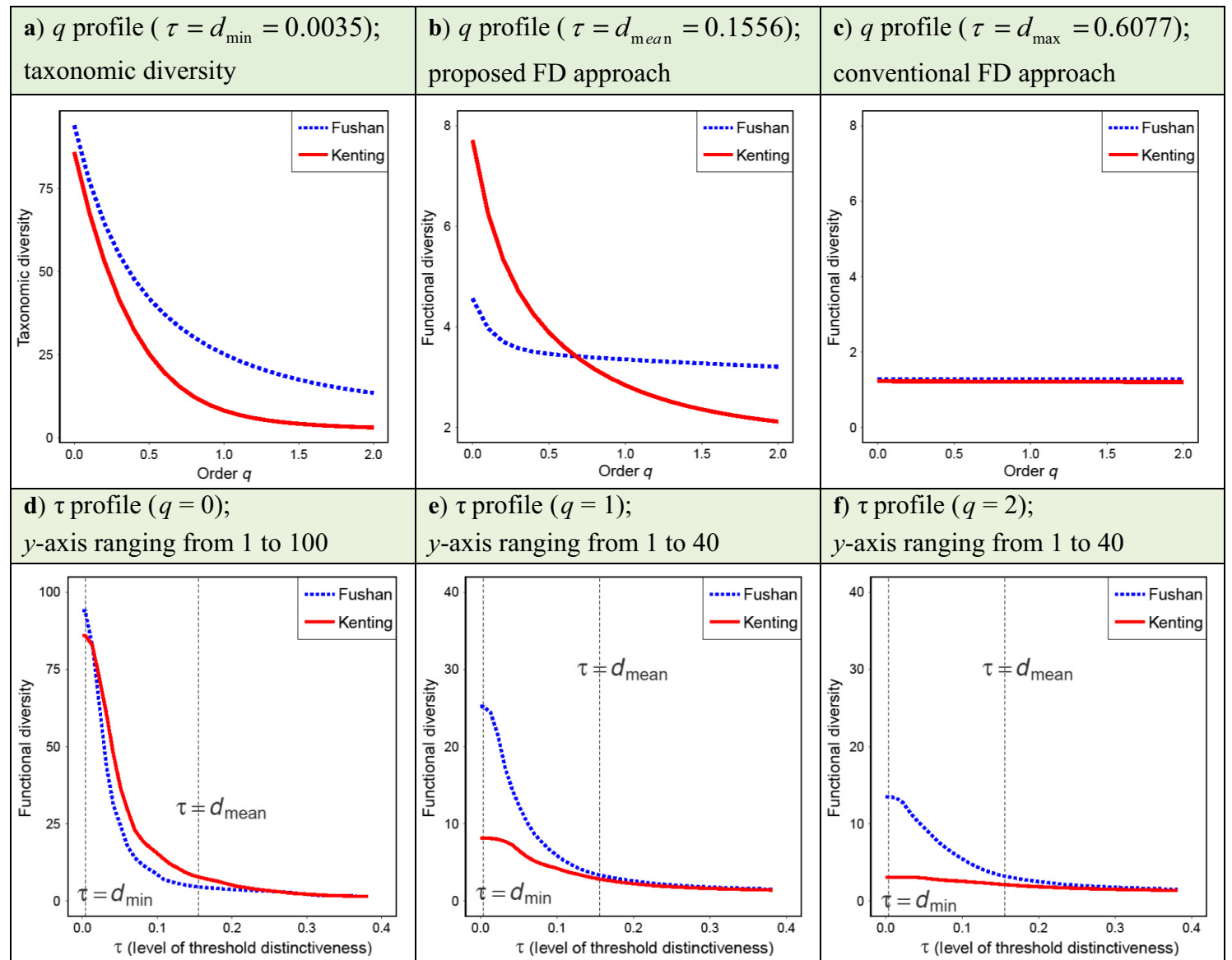

FIG. 5. The within-plot functional diversity profiles for two Forest Dynamic Plots in Taiwan (Fushan and Kenting) as a function of diversity order $q, 0 \leq q \leq 2$ (panels a, b, c), or as a function of threshold distinctiveness level $\tau, 0<\tau<0.4$ (panels d, e, f), based on species relative abundance data ( 10 ha for each plot). The two dotted vertical lines in the lower panels refer to two different levels of threshold distinctiveness: $d_{\min }(0.0035)$ and $d_{\text {mean }}(0.1556)$, the minimum and mean of the species-pairwise distances in the pooled assemblage. The curves are truncated at 0.4 as the diversity values remain at a constant when $\tau>0.4$, and thus, the functional diversity value for $d_{\max }(0.6077)$ for each plot is not shown.

In contrast, FS is a typhoon-prone forest that experiences a mean of 0.74 typhoon per year, i.e., on average, a typhoon three out of every four years (Lin et al. 2011). The high frequency of wind disturbance may select for species with pioneer-like leaf traits that adapt to high light and strong wind conditions. This strong environmental filtering effect may result in the higher functional evenness and lower functional richness despite the higher taxonomic richness found in FS.

The between-plot taxonomic Sørensen and Jaccard taxonomic dissimilarity values are relatively high for $0 \leq q \leq 2$, as only $7.1 \%$ species are shared and nearly all of the more common and dominant species are nonshared (Fig. 6a, b). By contrast, functional dissimilarity values based on the conventional approach are all close to zero, indicating no functional differentiation between the two plots. The curve based on our approach is between the two extremes and exhibits moderate dissimilarity. For example, the Sørensen functional dissimilarity (i.e., the mean proportion of non-shared functional groups in each plot) is 7.4\% $(q=0), 36.0 \%$ $(q=1)$, and $56.9 \%(q=2)$. The corresponding Jaccard functional dissimilarity (i.e., the proportion of nonshared functional groups in the pooled plot) is $13.7 \%$ $(q=0), \quad 36.0 \% \quad(q=1), \quad$ and $39.7 \% \quad(q=2) ;$ see Appendix S4: Table S2. Unlike the results shown in Fig. 4, where dissimilarity $q$ profiles generally decline with order $q$, here both the Sørensen and Jaccard functional dissimilarity $q$ profiles are monotonically increasing functions of $q$, signifying that it is the abundant species that mainly contribute to the functional differentiation between the two plots. This effect of abundant species is reflected by all three $\tau$ profile curves $(q=0,1$, and 2) in the two lower panels: the curve for $q=2$ lies above that for $q=1$, which lies above that for $q=0$. This finding is generally valid for any threshold level for both Sørensen and Jaccard functional dissimilarity measures (Fig. 6c, d). 


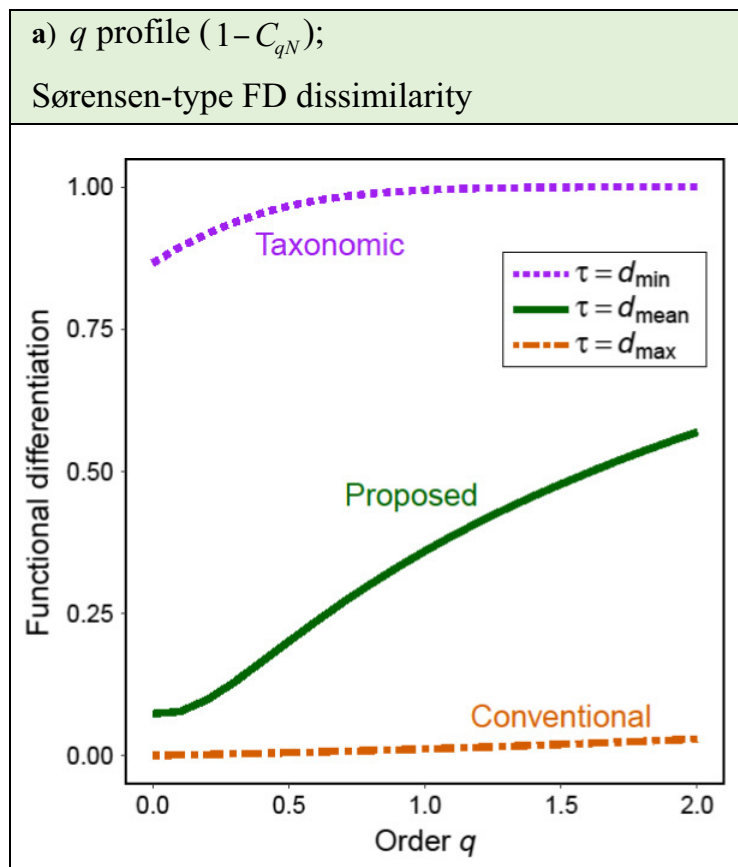

c) $\tau$ profile $\left(1-C_{q N}\right)$;

Sørensen-type FD dissimilarity

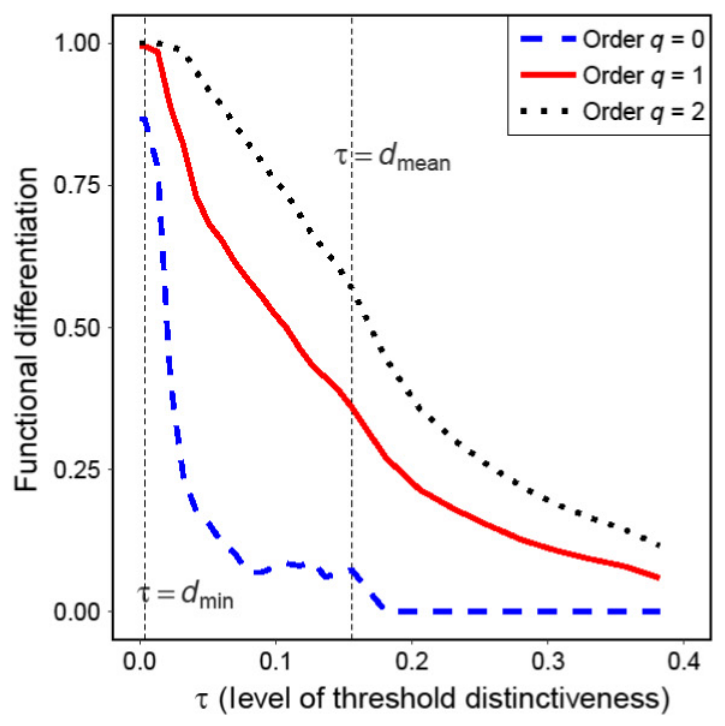

b) $q$ profile $\left(1-U_{q N}\right)$;

Jaccard-type FD dissimilarity

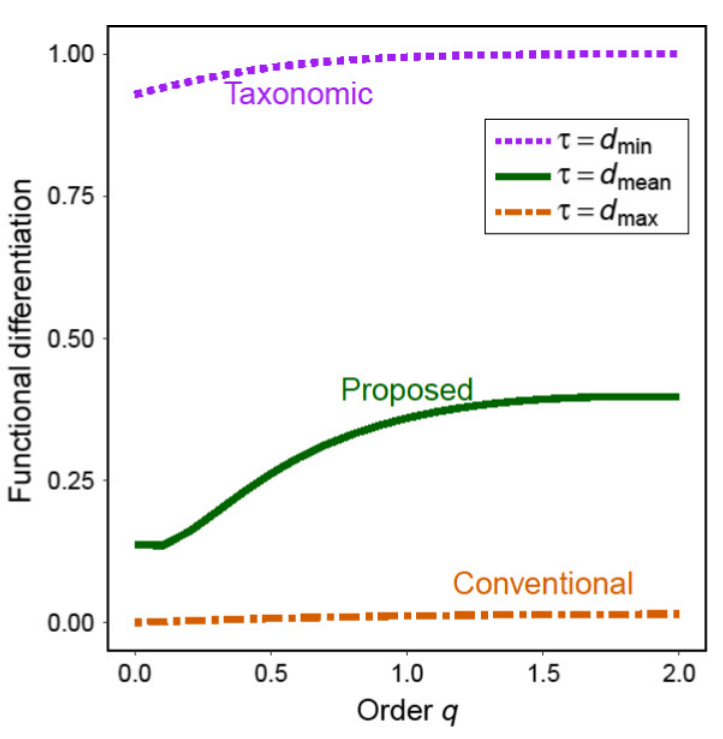

d) $\tau$ profile $\left(1-U_{q N}\right)$;

Jaccard-type FD dissimilarity

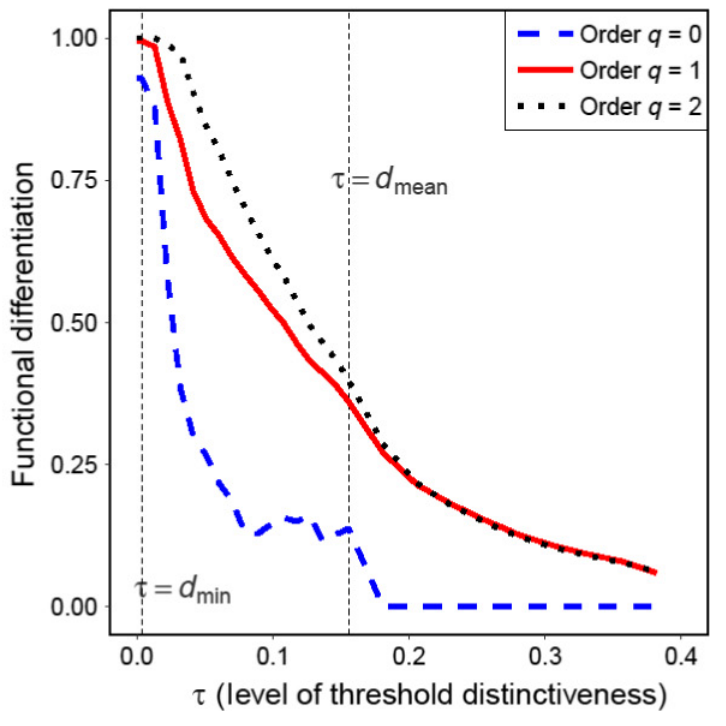

FIG. 6. The between-plot (a, c) Sørensen-type (Eq. 9a) and (b, d) Jaccard-type (Eq. 9b) functional differentiation/dissimilarity profile for two Forest Dynamics Plots in Taiwan (Fushan and Kenting) as a function of diversity order $q, 0 \leq q \leq 2$ (panels a, b), or as a function of threshold distinctiveness level $\tau, 0<\tau<0.4$ (panels $\mathrm{c}, \mathrm{d}$ ), based on species relative abundance data ( 10 ha for each plot). See Fig. 5 for the meaning of the vertical dotted lines in the lower panels and the values of $d_{\min }$ and $d_{\text {mean }}$. The curves are truncated at $\tau=0.4$, and thus, the dissimilarity value for $\tau=d_{\max }(0.6077)$ for each order of $q$ is not shown.

\section{ConcLusion AND Discussion}

In this paper, we have proposed a new class of distance-based functional diversity of order $q,{ }^{q} F D(\Delta(\tau))$ (in
Eqs. $4 \mathrm{a}-4 \mathrm{e}$ ), for a given level of threshold distinctiveness $\tau$ and the corresponding truncated matrix $\Delta(\tau)$ (Eq. $3 b$ ). The parameter $\tau$ specifies the effective distance between any two virtual functional groups in the reference 
assemblage. Our functional diversity quantifies the effective number of functionally equally distinct species (or virtual functional groups) at the specified level of $\tau$. The implementation of a threshold distinctiveness level is essential, as also emphasized in the threshold distinctiveness level subsection below.

Although a three-dimensional profile (Fig. 2a) can convey all the information related to our functional diversity, we advocate the use of two types of profiles that depict the equivalent number of species, first as a function of diversity order $q$ ( $q$ profile), and then as a function of threshold level $\tau$ ( $\tau$ profile); see Figs. 2b, c, 3 , and 5 for examples. Functional diversities of assemblages in which species are described using the same functional traits can be meaningfully ranked and compared on the basis of the two diversity profiles at the same threshold distinctiveness level $\tau$. In addition, the $q$ profile at $\tau=d_{\min }$ represents a taxonomic diversity profile based on Hill numbers; see Figs. 3a and 5a. The $q$ profile at $\tau=d_{\max }$ represents a profile based on the conventional species-equivalent formula (Eqs. $5 \mathrm{c}$ and $5 \mathrm{~d}$ ) that quantifies the effective number of maximally distinct species; see Figs. 3c and 5c. Our theory can be readily applied to explain why the conventional speciesequivalent formulas often yield low values in most functional diversity analyses. Functional diversity profiles may cross (e.g., Fig. 5b), reversing the ranking of assemblages at different values of $q$ and/or $\tau$; these reversals can convey important ecological information.

In practice, when there are many assemblages, such that profile comparison is challenging, we suggest comparing the functional diversities for $q=0,1$, and 2 computed at $\tau=d_{\text {mean }}$, which is the mean distance between any two individuals (including same-species pairs) taken from the pooled assemblage; see Appendix S4: Tables S1-S4 for examples. We could also consider other choices of $\tau$ values, such as the mean of individual $Q$ s over all assemblages or the mean distance between any two individuals excluding same-species pairs. Moreover, one could also use an integrated value over all plausible levels of $\tau$ such as the area under the curve (AUC) of a $\tau$ profile in a specified range (e.g., $[0,1]$ for the Gower distance, or $\left[d_{\min }, d_{\max }\right]$ for an unbounded distance metric). The value of the AUC quantifies an overall mean functional diversity for all possible threshold levels. The examples provided in Appendix S2: Tables S2 and S4 demonstrated that our proposed functional diversity computed at $\tau=d_{\text {mean }}$ yield rankings among assemblages highly consistent with those yielded by the AUC index for any fixed value of $q=0,1$, and 2 .

We also proposed a multiplicative partitioning scheme based on our attribute-diversity framework and derived the formulas for functional alpha, beta, and gamma diversities. The beta component can be monotonically transformed to Sørensen- or Jaccard-type functional (dis)similarity measures. The interpretations of our alpha, beta, gamma, and (dis)similarity measures are generally similar to those in taxonomic diversity. The only difference is that all species-equivalents in functional diversity are assumed to be equally distinct at the specified level of threshold distinctiveness. Unlike the restrictions on distance matrices for a proper decomposition in the conventional approach, our proposed gamma is never less than the corresponding alpha for any type of distance matrix. We have also extended the two types of profiles ( $\tau$ profile and $q$ profile) to the associated dissimilarity profiles; see Figs. 4 and 6 for examples. All the proposed profiles provide informative measures to quantify and compare functional differentiation across multiple sets of assemblages.

\section{The threshold distinctiveness level}

We use a simple example to emphasize that it is essential to introduce the additional parameter of a threshold distinctiveness level in distance-based functional diversity. Assume that each assemblage (I and II) includes four equally abundant species, and there are no shared species between the two assemblages.

Case a.-We only consider Assemblage I in which any two different species have a constant distance of 2. Then the conventional functional diversity (Eqs. $5 \mathrm{c}$ and $5 \mathrm{~d}$ ) for Assemblage I is 4 for all $q \geq 0$.

Case b.-Suppose in the comparison we add Assemblage II in which two different species have a constant distance of 8 . If we normalize all distances by dividing each distance by the "global" maximum, which is 8 , then the conventional functional diversity for Assemblage II is 4 , whereas the conventional functional diversity for Assemblage I is changed to 1.23 for all $q \geq 0$; see Appendix S2 for computational details.

It is evident from the above example that one cannot compare diversity values from different cases; otherwise Assemblage I in Case a would have exactly the same diversity as Assemblage II in Case b, which is obviously a wrong conclusion because the all the non-zero speciespairwise distances in Assemblage II is higher than those of Assemblage I. This example also reveals that the conventional functional diversity of Assemblage I depends on the presence/absence of Assemblage II. Thus, one additional parameter is needed to distinguish the two different functional diversity values of Assemblage I in the two cases. In fact, it can be readily observed that applying the conventional approach as described above is equivalent to setting different threshold levels in our framework: a level $\tau=2$ in Case a with another level $\tau=8$ in Case $b$. That is, the two functional diversities of Assemblage I in the two different cases can be interpreted as (in an effective sense): there are four equally distinct species with pairwise distance $\geq 2$ for differentspecies pairs in Case a; whereas, in Case b, there are 1.23 equally distinct species with pairwise distance $\geq 8$ for different-species pairs. Using a parameter of threshold distinctiveness level, as implemented in our framework, 
readily solves this problem. In other words, for Assemblage $\mathrm{I}$, the $\tau$ profile value at $\tau=2$ is 4 (the conventional functional diversity in Case a) whereas the value at $\tau=8$ is 1.23 (the conventional functional diversity in Case b). The use of a $\tau$ profile means that the conventional diversity is always incorporated into our proposed $\tau$ profile.

Moreover, note in the above conventional approach, one can only obtain the effective number of species for the maximum distance or global maximum distance, i.e., 2 in Case a and 8 in Case b. As our theory predicts, the resulting diversity values are necessarily low in most applications. Such low diversity is a nearly inescapable consequence of setting a very high level of threshold distinctiveness. Implementing an adjustable $\tau$ parameter allows for the functional diversity of all assemblages to be evaluated and thus compared at any desired threshold level.

Based on our proposed $\tau$ profiles for the two assemblages, our ranking of them implies that they are equally diverse when $0<\tau \leq 2$, but Assemblage II is more diverse when $\tau>2$. Our functional diversity profile for any assemblage is independent of the presence/absence of other assemblages involved in the comparison. This example highlights the importance of implementing a threshold distinctiveness level and using a $\tau$ profile; it also demonstrates that two assemblages can be meaningfully ranked only at the same level of threshold distinctiveness. The functional diversities of two assemblages computed using different levels of threshold distinctiveness cannot be used to rank assemblages. See Appendix S2 for more examples.

\section{Raw abundance data vs. within-assemblage relative abundance data}

All functional diversity formulas (Eqs. 4a-4e) account only for species relative abundances. Thus, the two types of data (raw abundances and relative abundances) give identical functional diversity in a single assemblage. However, when there are multiple assemblages, the two types of data lead to different functional alpha, beta and gamma diversities, because the raw abundances could yield different total abundances among assemblages while the relative abundances always sum to 1 (i.e., all assemblages have the same weight for diversity computation).

The choice of data type should be determined by the question under investigation and the type of sampling utilized in the study. Generally, if sampling proportions or fractions in all assemblages are equal (sampling proportion is defined as the ratio of sample size to assemblage size), then both species raw abundances and relative abundances can be used in our procedure to assess functional dissimilarity among assemblages; in this case, the total of species raw abundances in each assemblage is expected to reflect the ecological importance of an assemblage. If the sampling proportions in the assemblages are uneven (e.g., sampling 100 trees per assemblage, regardless of their actual density), then species raw abundances cannot be compared across assemblages, and only species relative abundances can be used. Unless sampling is complete or assemblage size is known, the sampling proportion is often unknown. In our presentation, parallel derivations hold by replacing $n_{i k}$ (the number of individuals of the $i$ th species in the $k$ th assemblage) by $p_{i k}=n_{i k} / n_{+k}$ (within-assemblage relative abundance of the $i$ th species in the $k$ th assemblage) and making other replacements accordingly in all steps. See Appendix S4: Tables S3 and S4 for details and numerical comparisons.

\section{Three components of functional diversity}

As stated in Introduction, previous authors have proposed that trait-based functional diversity should include three primary components: functional richness, functional evenness, and functional divergence. In ordinary Hill numbers $\left({ }^{q} D\right.$, defined in Eq. 1a), zero-order diversity represents species richness. For any $q>0$, the normalized slope of the diversity profile in the range $[0,1]$ is expressed as $\left({ }^{q} D-1\right) /\left({ }^{0} D-1\right)$, which quantifies the evenness among species relative abundances (Jost 2010). Alternatively, the slope of the Réyni-entropy (the logarithm of Hill number) profile, $\log \left({ }^{q} D\right) / \log \left({ }^{0} D\right)$, can also be used to quantify species evenness.

Recall that our functional diversity of order $q\left({ }^{q} F D(\Delta\right.$ $(\tau))$, Eq. 4a) is simply the Hill number of the same order for an assemblage in which there are $S$ sub-assemblages: the $i$ th sub-assemblage consists of $v_{i}(\tau)=n_{i} / a_{i}(\tau)$ functional groups, each with group "relative" abundance $a_{i}(\tau) / n_{+}, i=1,2, \ldots, S$. By analogy with Hill numbers, we can quantify functional richness by the order-zero measure, ${ }^{0} F D(\Delta(\tau))$, which is the total number of functional groups for a specified threshold level $\tau$. As explained in Properties of our functional diversity, our zero-order measure generally depends on species abundance. However, if we set all relative abundances to be $1 / S$ (i.e., giving the same weight to all species of an assemblage), functional richness is independent of species abundances. Our indices could thus be applied when only species presence/ absence information is available (e.g., large-scale studies looking for differences in species composition).

In a similar manner, functional evenness can be measured by the two normalized slopes, $\left[{ }^{q} F D(\Delta(\tau))-1\right] /$ $\left[{ }^{0} F D(\Delta(\tau))-1\right]$ and $\log \left[{ }^{q} F D(\Delta(\tau))\right] / \log \left[{ }^{0} F D(\Delta(\tau))\right]$ for any $q>0$. These measures quantify the extent of evenness of functionally indistinct set abundances $\left(a_{1}(\tau), a_{2}(\tau), \ldots a_{S}(\tau)\right)$, weighted by the attribute contribution, $v_{i}(\tau)$, of each species. Mason et al. (2005) defined functional divergence as the extent of the spread of trait values with respect to the center of a functional space, weighted by species abundance; it roughly monotonically increases with the abundance-weighted mean species pairwise distance (e.g., quadratic entropy), or equivalently, monotonically increases with the abundance-weighted functional groups (e.g., see Eq. 4e for $q=2$ ). For any order $q>0$, the proposed abundanceweighted functional diversity, ${ }^{q} F D(\Delta(\tau))$, quantifies the 
effective number of abundance-weighted functional groups (with the parameter $q$ controlling the weighting of species abundance), and thus can be used to quantify functional divergence.

\section{Intraspecific variability}

Although we assume no intraspecific variability for species traits (i.e., the diagonal elements of the distance matrix are 0 ), our approach potentially can be extended to instances when trait values are available at the individual level. For example, the distance for a pair of species (including the same species pair) could be defined as the mean of the distances of all different individual pairs. If individuals of different sexes or ages have contrasting functional trait values, because of sexual dimorphism or ontogenetic shift, it is possible to account for the mean trait values of such functional entities (e.g., age classes, sexes) for computing functional diversity indices. Parallel derivations of Eqs. $3 \mathrm{c}$ and $3 \mathrm{~d}$ can be generalized to incorporate intraspecific variability. Nevertheless, more research is needed along this path.

\section{Statistical issues}

Like species diversity, our proposed functional diversity based on sampling data depends on sampling effort and sampling completeness. Chao et al. (2014b) developed rarefaction and extrapolation formulas for Hill numbers. The corresponding formulas for the proposed functional diversity profile will be reported elsewhere to facilitate legitimate comparison among multiple assemblages based on incomplete sampling data.

\section{A unified profile-based approach to taxonomic, phylogenetic, and functional diversity}

There is a growing consensus that the $q$ profile of Hill numbers (which depicts Hill numbers as a function of the diversity order $q$ ) completely characterizes taxonomic diversity in an assemblage (Ellison 2010). In this paper, we have advocated the use of two profiles ( $q$ profile and $\tau$ profile) to characterize functional diversity. The $q$ profile for $\tau=d_{\min }$ is identical to the taxonomic diversity profile; the leftmost level of each $\tau$ profile (i.e., when $\tau$ is less than or equal to the minimum distance) also represents taxonomic diversity. Although our approach can be applied to pairwise phylogenetic distances, we recommend the use of tree-based phylogenetic diversity (Chao et al. 2010, Chiu et al. 2014), because that measure integrates over all relationships. For a tree-based measure, all species interrelations not only for a pair of species, but also for every possible combination of three species, four species, etc. can be incorporated, whereas the current functional diversity based on a pairwise distance matrix only considers the relations between any two species. In the special case that the phylogenetic tree is ultrametric and the phylogenetic distance matrix is defined as the corresponding cophenetic distance matrix, our pairwise-distance-based functional diversity of $q=2$ is identical to the Chao et al.'s (2010) tree-based phylogenetic diversity; see Appendix S5 for details. However, the two approaches lead to different results for any other value $q \neq 2$. A numerical example is given in Appendix S5: Table S1 to show the differences.

Chao et al. (2010) proposed two profiles ( $q$ profile and $T$ profile) for their tree-based phylogenetic diversity where the time parameter $T$ means that the phylogenetic diversity is computed for the time period from the past $T$ years to the present. Here, the time parameter $T$ in phylogenetic diversity plays exactly the same role as the level $\tau$ in functional diversity. That is, any two species with cophenetic distance $\geq T$ are treated as phylogenetically equally divergent in the specified time period; see the illustrative figures in Appendix S5. The $q$ profile for $T=0$ is identical to the taxonomic diversity profile; the leftmost level of each $T$ profile (i.e., when $T$ tends to zero) also represents taxonomic diversity. Thus, these profile-based perspectives provide a unified approach to the three dimensions of biodiversity.

\section{Biogeographical gradient in functional diversity}

Assessing functional diversity has been mostly applied on local communities to disentangle assembly rules, including biotic competition and environmental filtering, as well as to measure relationship between biodiversity and ecosystem functioning. For a decade, an increasing effort to collect trait values on hundreds to thousands of species has permitted the emergence of functional biogeography, which aims to map patterns of functional diversity on a large scale (from ecoregions to the world), to analyze their determinants and to predict their trends under global change (Violle et al. 2014). The proposed set of indices offers an opportunity to comprehensively assess the spatial gradients in functional alpha- and beta-diversity, and to compare them with those for taxonomic and phylogenetic biodiversities.

\section{ACKNOWLEDGMENTS}

The authors thank the Subject Matter Editor (Helmut Hillebrand), Jason Bragg, Marc Cadotte, Robert Colwell, William Cornwell, Lou Jost, Alexey Ryabov, Samuel Scheiner, and Peter Smouse for providing very thoughtful and helpful comments and discussions on topics related to the subject of this paper. We also thank S.-H. Wu from Taiwan Forestry Research Institute for her contribution in collecting the Kenting data, and $\mathrm{Yu}-Y u n$ Chen for data management of the $\mathrm{Fu}-$ Shan data. This work is supported by the Taiwan Ministry of Science and Technology under Contract 106-2628-M-007-01 (for A. Chao), 104-2118-M-002-008-MY3 (for C.-H. Chiu), and NSC- 99-2621-B-259-002-MY3 (for I.-F. Sun). The Fushan Forest Dynamics Plot is supported by the Taiwan Forestry Bureau and the Taiwan Forestry Research Institute. We thank the staff at the Fushan Research Center for providing logistic support. 


\section{Literature Cited}

Botta-Dukát, Z. 2018. The generalized replication principle and the partitioning of functional diversity into independent alpha and beta components. Ecography 41:40-50.

Cadotte, M. W., K. Carscadden, and N. Mirotchnick. 2011. Beyond species: functional diversity and the maintenance of ecological processes and services. Journal of Applied Ecology 48:1079-1087.

Champely, S., and D. Chessel. 2002. Measuring biological diversity using Euclidean metrics. Environmental and Ecological Statistics 9:167-177.

Chao, A., and C.-H. Chiu. 2016. Bridging the variance and diversity decomposition approaches to beta diversity via similarity and differentiation measures. Methods in Ecology and Evolution 7:919-928.

Chao, A., C.-H. Chiu, and L. Jost. 2010. Phylogenetic diversity measures based on Hill numbers. Philosophical Transactions of the Royal Society B 365:3599-3609.

Chao, A., C.-H. Chiu, and L. Jost. 2014a. Unifying species diversity, phylogenetic diversity, functional diversity, and related similarity and differentiation measures through Hill numbers. Annual Review of Ecology, Evolution, and Systematics 45:297-324.

Chao, A., N. J. Gotelli, T. Hsieh, E. L. Sander, K. Ma, R. K. Colwell, and A. M. Ellison. 2014b. Rarefaction and extrapolation with Hill numbers: a framework for sampling and estimation in species diversity studies. Ecological Monographs 84:45-67.

Chiu, C.-H., and A. Chao. 2014. Distance-based functional diversity measures and their decomposition: a framework based on Hill numbers. PLoS ONE 9:e100014.

Chiu, C.-H., L. Jost, and A. Chao. 2014. Phylogenetic beta diversity, similarity, and differentiation measures based on Hill numbers. Ecological Monographs 84:21-44.

Cornwell, W. K., D. W. Schwilk, and D. D. Ackerly. 2006. A trait-based test for habitat filtering: Convex hull volume. Ecology 87:1465-1471.

de Bello, F., S. Lavorel, S. Díaz, R. Harrington, J. H. Cornelissen, R. D. Bardgett, M. P. Berg, P. Cipriotti, C. K. Feld, and D. Hering. 2010. Towards an assessment of multiple ecosystem processes and services via functional traits. Biodiversity and Conservation 19:2873-2893.

Devictor, V., D. Mouillot, C. Meynard, F. Jiguet, W. Thuiller, and N. Mouquet. 2010. Spatial mismatch and congruence between taxonomic, phylogenetic and functional diversity: the need for integrative conservation strategies in a changing world. Ecology Letters 13:1030-1040.

Ellison, A. M. 2010. Partitioning diversity. Ecology 91:19621963.

Garnier, E., J. Cortez, G. Billès, M.-L. Navas, C. Roumet, M. Debussche, G. Laurent, A. Blanchard, D. Aubry, and A. Bellmann. 2004. Plant functional markers capture ecosystem properties during secondary succession. Ecology 85:2630-2637.

Gower, J. C. 1971. A general coefficient of similarity and some of its properties. Biometrics 27:857-871.

Gregorius, H.-R., and E. Kosman. 2017. On the notion of dispersion: from dispersion to diversity. Methods in Ecology and Evolution 8:278-287.

Hill, M. 1973. Diversity and evenness: A unifying notation and its consequences. Ecology 54:427-432.

Hillebrand, H., et al. 2018. Biodiversity change is uncoupled from species richness trends: Consequences for conservation and monitoring. Journal of Applied Ecology 55:169-184.

Hooper, D. U., et al. 2005. Effects of biodiversity on ecosystem functioning: A consensus on current knowledge. Ecological Monographs 75:3-35.
Jost, L. 2010. The relation between evenness and diversity. Diversity 2:207-232.

Legendre, P., and L. Legendre. 2012. Numerical ecology. Third English edition. Elsevier, Amsterdam, the Netherlands.

Leinster, T., and C. A. Cobbold. 2012. Measuring diversity: the importance of species similarity. Ecology 93:477-489.

Lin, T.-C., S. P. Hamburg, K.-C. Lin, L.-J. Wang, C.-T. Chang, Y.-J. Hsia, M. A. Vadeboncoeur, C. M. Mabry McMullen, and C.-P. Liu. 2011. Typhoon disturbance and forest dynamics: lessons from a northwest pacific subtropical forest. Ecosystems 14:127-143.

Maire, E., G. Grenouillet, S. Brosse, and S. Villéger. 2015. How many dimensions are needed to accurately assess functional diversity? A pragmatic approach for assessing the quality of functional spaces. Global Ecology and Biogeography 24:728740.

Mason, N. W. H., D. Mouillot, W. G. Lee, and J. B. Wilson. 2005. Functional richness, functional evenness and functional divergence: the primary components of functional diversity. Oikos 111:112-118.

Mason, N. W. H., F. de Bello, D. Mouillot, S. Pavoine, and S. Dray. 2013. A guide for using functional diversity indices to reveal changes in assembly processes along ecological gradients. Journal of Vegetation Science 24:794-806.

Mouchet, M., F. Guilhaumon, S. Villéger, N. W. Mason, J. A. Tomasini, and D. Mouillot. 2008. Towards a consensus for calculating dendrogram-based functional diversity indices. Oikos 117:794-800.

Mouchet, M. A., S. Villéger, N. W. Mason, and D. Mouillot. 2010. Functional diversity measures: an overview of their redundancy and their ability to discriminate community assembly rules. Functional Ecology 24:867-876.

Mouillot, D., S. Villéger, M. Scherer-Lorenzen, and N. W. H. Mason. 2011. Functional structure of biological communities predicts ecosystem multifunctionality. PLoS ONE 6:e17476.

Mouillot, D., N. A. Graham, S. Villéger, N. W. H. Mason, and D. R. Bellwood. 2013. A functional approach reveals community responses to disturbances. Trends in Ecology and Evolution 28:167-177.

Naeem, S., J. E. Duffy, and E. Zavaleta. 2012. The functions of biological diversity in an age of extinction. Science 336:14011406.

Petchey, O. L., and K. J. Gaston. 2002. Functional diversity (FD), species richness and community composition. Ecology Letters 5:402-411.

Rao, C. R. 1982. Diversity and dissimilarity coefficients: a unified approach. Theoretical Population Biology 21:24-43.

Ricotta, C. 2005. A note on functional diversity measures. Basic and Applied Ecology 6:479-486.

Ricotta, C., and A. Acosta. 2014. On the functional diversity of partially distinct species: some theory and a practical example. Community Ecology 15:205-211.

Ricotta, C., and L. Szeidl. 2006. Towards a unifying approach to diversity measures: bridging the gap between the Shannon entropy and Rao's quadratic index. Theoretical Population Biology 70:237-243.

Ricotta, C., and L. Szeidl. 2009. Diversity partitioning of Rao's quadratic entropy. Theoretical Population Biology 76:299-302.

Scheiner, S. M., E. Kosman, S. J. Presley, and M. R. Willig. 2017. Decomposing functional diversity. Methods in Ecology and Evolution 8:809-820.

Seibold, S., R. Brandl, J. Buse, T. Hothorn, J. Schmidl, S. Thorn, and J. Müller. 2015. Association of extinction risk of saproxylic beetles with ecological degradation of forests in Europe. Conservation Biology 29:382-390.

Stuart-Smith, R. D., A. E. Bates, J. S. Lefcheck, J. E. Duffy, S. C. Baker, R. J. Thomson, J. F. Stuart-Smith, N. A. Hill, S. J. 
Kininmonth, and L. Airoldi. 2013. Integrating abundance and functional traits reveals new global hotspots of fish diversity. Nature 501:539-542.

Su, S.-H., C. H. Chang-Yang, C. L. Lu, C. C. Tsui, T. T. Lin, C. L. Lin, W. L. Chiou, L. H. Kuan, Z. S. Chen, and C. F. Hsieh. 2007. Fushan subtropical forest dynamics plot: tree species characteristics and distribution patterns. Taiwan Forestry Research Institute, Taipei, Taiwan.

Swenson, N. G., et al. 2012. Phylogenetic and functional alpha and beta diversity in temperate and tropical tree communities. Ecology 93:112-125.

Thorn, S., C. Bässler, H. Bußler, D. B. Lindenmayer, S. Schmidt, S. Seibold, B. Wende, and J. Müller. 2016. Bark-scratching of storm-felled trees preserves biodiversity at lower economic costs compared to debarking. Forest Ecology and Management 364:10-16.

Tilman, D., J. Knops, D. Wedin, P. Reich, M. Ritchie, and E. Siemann. 1997. The influence of functional diversity and composition on ecosystem processes. Science 277:1300-1302.
Villéger, S., N. W. Mason, and D. Mouillot. 2008. New multidimensional functional diversity indices for a multifaceted framework in functional ecology. Ecology 89:2290-2301.

Villéger, S., J. R. Miranda, D. F. Hernandez, and D. Mouillot. 2012. Low functional $\beta$-diversity despite high taxonomic $\beta$ diversity among tropical estuarine fish communities. PLoS ONE 7:e40679.

Violle, C., P. B. Reich, S. W. Pacala, B. J. Enquist, and J. Kattge. 2014. The emergence and promise of functional biogeography. Proceedings of the National Academy of Sciences USA 111:13690-13696.

Weiher, E. 2011. A primer of trait and functional diversity. Pages 175-193 in A. Magurran and B. J. McGill, editors. Biological diversity: frontiers in measurement and assessment. Oxford University Press, Oxford, UK.

Wu, S.-H., Z.-Y. Hseu, Y.-T. Shih, I.-F. Sun, H.-H. Wang, and Y.-C. Sen. 2011. Kenting karst forest dynamics plot: tree species characteristics and distribution patterns. Taiwan Forestry Research Institute, Taipei, Taiwan.

\section{SUPPORTING INFORMATION}

Additional supporting information may be found online at: http://onlinelibrary.wiley.com/doi/10.1002/ecm.1343/full

\section{Data Availability}

Data used in this paper are available in Zenodo: https://doi.org/10.5281/zenodo.1465359. 